\title{
Spatio-Temporal Evolution of Harmonic Disturbances on Laminar, Non-Premixed Flames: Measurements and Analysis
}

\author{
Nicholas Magina, William Steele, Benjamin Emerson, Timothy Lieuwen \\ School of Aerospace Engineering, Georgia Institute of Technology, \\ Atlanta, Georgia 30332-0150, USA \\ Contact Information: \\ Mailing Address: Ben T. Zinn Combustion Lab \\ 635 Strong St NW \\ Atlanta, GA, 30318, USA \\ Fax: +1-404-463-0888 \\ Email: narthurm@gatech.edu
}




\begin{abstract}
This paper analyzes the dynamics of harmonically forced, non-premixed flames, both experimentally and computationally. Prior theory has made a number of predictions on how wrinkles on non-premixed flamelets are excited by flow disturbances, convect axially, and evolve in disturbance magnitude, including setting up interference patterns in wrinkle disturbance magnitude. The objective of this study was to obtain measurements from forced flames to determine if these features are present, and to compare the gain/phase of these wrinkles with predictions using the measured velocity field as inputs. High speed PIV data was taken on a coflowing methane-air diffusion flame, equipped with speakers for harmonic forcing, over a variety of flow velocities, forcing frequencies, and forcing amplitudes. These measured velocity fields were used as inputs to a $z$-equation solver, and the resulting space-time dynamics of iso- $z$ surfaces were extracted from the $z$ field solutions. Both experimental and numerical results show that flame wrinkles propagate axially at the mean flow velocity, a result consistent with previous analytical findings. These wrinkles start with near zero magnitude at the fuel tube lip and grow with downstream distance, until peaking at some axial location. Further downstream, the wrinkle magnitude modulates, indicative of interference effects which have been previously predicted in analytical studies.
\end{abstract}

\title{
Keywords
}

Non-premixed flame, flame dynamics, flame transfer function, flame response, velocity coupled response, combustion instabilities

\section{Nomenclature}

Q) Diffusion coefficient

Fr $\quad$ Froude number 


\begin{tabular}{|c|c|}
\hline$L_{f}$ & Flame length \\
\hline$M$ & Molecular weight \\
\hline$\odot$ & Reference velocity spatial coordinate, at $x=0$ and $y=R_{w} / 2$ \\
\hline$P$ & Pressure \\
\hline $\mathrm{Pe}$ & Peclet number \\
\hline$R_{f}, R_{w}$ & Radial distance to fuel tube lip and confining wall, respectively \\
\hline Sk & Stokes number \\
\hline St & Strouhal number defined as $S t=f R_{f} / U_{0}$ \\
\hline$T$ & Temperature \\
\hline$T_{a}, T_{f}$ & Ambient and adiabatic flame temperature, respectively \\
\hline$U_{x}, U_{r}, U_{c}$ & Axial flow, radial flow, and wrinkle convection velocity, respectively \\
\hline$U_{0, \mathrm{des}}$ & Targeted fuel and oxidizer axial flow velocity \\
\hline$V$ & Atomic diffusion volume \\
\hline$z$ & Mixture fraction \\
\hline$Z_{s t}$ & Stoichiometric mixture fraction value \\
\hline$z^{*}$ & Mixture fraction value at which mean mixture properties were extracted from \\
\hline$f, \omega$ & Forcing and angular frequencies, respectively \\
\hline$f_{b}, f_{f}$ & Buoyant natural instability and forcing frequencies, respectively \\
\hline$x, y$ & Axial and radial coordinate \\
\hline$\varepsilon$ & Small perturbation parameter \\
\hline$\theta_{0}$ & Local angle of unforced flame surface with respect to axial coordinate \\
\hline$\xi$ & Radial flame position \\
\hline$\varphi_{c}$ & Stoichiometric mass ratio of oxidizer to fuel \\
\hline
\end{tabular}


()$_{0},()_{1} \quad$ Mean/steady state and fluctuating components respectively

( ) Fourier Transform

\section{Introduction}

This paper describes measurements and analysis of the space-time evolution of disturbances on harmonically forced, laminar, non-premixed flames. Propagating waves are excited on iso-scalar surfaces when flamelets are disturbed, in addition to disturbing the internal structure of the flamelet. The objective of this study is to experimentally characterize the excitation and evolution of these disturbances, and to compare these results to those predicted utilizing the measured unsteady flow field as model inputs. This problem arises prominently during combustion instabilities, where narrowband flow disturbances excite the flame, leading to oscillations in the heat release which are often destructive to the system.

A significant experimental and modeling literature exists on the response of premixed flames to harmonic flow disturbances [1-5]. Measurements have experimentally characterized both the local space-time dynamics of wrinkles on laminar flames $[6,7]$, as well as the spatially integrated heat release [8]. These measurements show that wrinkles are excited at the flame stabilization point and locations of spatial non-uniformities in disturbance velocity, and subsequently convect down the flame. The response of the flame at a given point in space and time is non-local - i.e., it is not only a function of the local disturbance, but is, rather, a convolution of disturbances excited at upstream locations at appropriate convective time delays. For these reasons, a variety of interference behaviors and spatially nonmonotonic variations in flame wrinkle magnitude occur [9]. Similar analytical studies of flame wrinkling behavior have also been performed in harmonically excited turbulent flames. In this case, the flame's local wrinkling and heat release consists of both the narrowband excitation, as well as 
broadband turbulent fluctuations - additionally these two types of disturbances nonlinearly interact with each other. For example, the harmonic forcing modulates the phase-averaged turbulent burning velocity, and the turbulent flame disturbances increase the rate of destruction of harmonic flame wrinkles [10, 11]. A particularly large literature has developed for global heat release response of turbulent flames to harmonic forcing, referred to as the heat release transfer function [12, 13]. Indeed, many industrial companies now possess capabilities for measuring flame transfer functions in high pressure, high flow facilities in order to screen their designs for instabilities.

There is significantly less literature on forced, non-premixed flames, particularly on the formation and evolution of wrinkles on the flame. Experimental efforts have elucidated some of the natural flame dynamics, such as flicker characteristics or buoyancy-related instabilities, and flow field characterization [14-18]. The impact of forcing on the flames emissive properties and soot generation/suppression has also been investigated [19-21]. One study reported on the flame wrinkle evolution characteristics of nonpremixed flames, including wrinkle magnitude as a function of axial position for unforced flickering flames [22]. We are not aware of any experimental studies of the space-time dynamics of flame wrinkles on forced, non-premixed flames, although some work has reported on the spatially integrated heat release and flame transfer function dynamics [23, 24]. The limited numerical results which do exist for the forced problem do not specifically focus on the unsteady wrinkle dynamics, but rather investigate advanced numerical approaches and flame shape [25].

A number of recent analytical studies have also been reported for forced, non-premixing flames, considering the space-time wrinkle dynamics, the space-time heat release dynamics, and the larger thermoacoustic system behavior [26-31]. These studies have analyzed this problem within the infinite reaction rate, $z$-equation formulation for the mixture fraction. This problem admits analytical solutions for certain types of imposed mean and fluctuating velocity fields (e.g., bulk forcing, convecting disturbance fields, and so forth), and constant diffusion coefficients. For example, Magina et al. [30] 
developed the following explicit solution for the fluctuating flame position, $\xi_{1, n}(x, t)$, of a non-premixed flames exposed to spatially uniform, axial flow oscillations:

$$
P e>>1 \quad \frac{\xi_{1, n}(x, t)}{R_{f}}=\frac{i \varepsilon \exp [-i \omega t]}{2 \pi S t} \sin \theta_{0}(x)\left[1-\exp \left[2 \pi i S t \frac{x}{R_{f}}\right] \exp \left[-\frac{4 \pi^{2} S t^{2}}{P e} \frac{x}{R_{f}}\right]\right]+O\left(\frac{1}{P e^{2}}\right)
$$

This expression is led by a group of terms which describe the wave magnitude and harmonic time dependence, revealing the low-pass filter characteristic of flame wrinkle amplitude, followed by a mean flame angle dependence. The bracketed term describes propagation of wrinkles along the reaction sheet. The leading order expansion of this expression, $1-\exp \left(i \omega x / U_{o}\right)$, shows how flame wrinkles propagate without dissipation and non-dispersively in the $P e \rightarrow \infty$ limit, convecting axially at the mean flow velocity. Local maxima and minima in flame wrinkle amplitude arise through interference between these two terms. The $\mathrm{O}(1 / P e)$ term describes the leading order decay of wrinkles.

While theory has predicted the excitation and propagation of these wrinkles on the flamelet, no comparable measurements or comparisons of the theories leading to expressions like Eq.(1) with experiments have been performed. Such comparisons are complicated by the fact that in real flames with temperature gradients, more complex shear flows, and convecting vortical disturbances, the evolution of flame wrinkles is more complex than the model disturbance profiles used in the theoretical analyses. Motivated by the above studies, the objective of this paper is to experimentally characterize the gain and phase characteristics of the flame wrinkle behavior, using actual measured mean and disturbance velocities as inputs to the governing flame dynamics equations in order to predict the flame response and to compare the axial evolution of these disturbances with theory. While a similar modeling effort has been done for premixed flames that used measured velocity fields as inputs to the flame dynamics equations $[6,7]$, we are not aware of a prior study of this nature for the non-premixed flame case. 


\section{Experimental Facility and Diagnostics}

Figure 1 shows the experimental facility and diagnostic setup used in this study. The key components of the facility are the fuel and air conditioning sections, combustor section, forcing section, and exhaust. Natural gas, $\sim 98 \% \mathrm{CH}_{4}$ with the balance consisting of higher hydrocarbons and inerts, passes through an 25psi system regulator and flows up the central fuel tube, of inner radius $R_{f}=4.50 \mathrm{~mm}$ and $0.254 \mathrm{~mm}$ wall thickness. Non-preheated air passes through a 75psi line regulator/filter and flows up a coflowing annular passage, of inner radius $R_{w}=4.0 \mathrm{~cm}$ and $5.0 \mathrm{~mm}$ wall thickness, that surrounds the fuel tube port outside diameter. The air passes through a hexagonal honeycomb flow straightener, with $4.78 \mathrm{~mm}$ apothem and nominal wall thickness of $0.8 \mathrm{~mm}, 1.10 \mathrm{~m}$ upstream of the burner outlet. Timeaveraged air and fuel volumetric fluxes (i.e., spatially averaged velocities) were matched to within $1 \%$ in order to minimize shear layer strength, so that the dominant source of excitation of the flame was from the acoustic forcing, and not the acoustically excited shear layers. The facility was operated at velocities of $20-90 \mathrm{~cm} / \mathrm{s}$. This velocity range and fuel (air) diameter correspond to Froude numbers in the range of 0.95-4.3, based upon $F r=U_{x} / \sqrt{g R_{f}}$. 


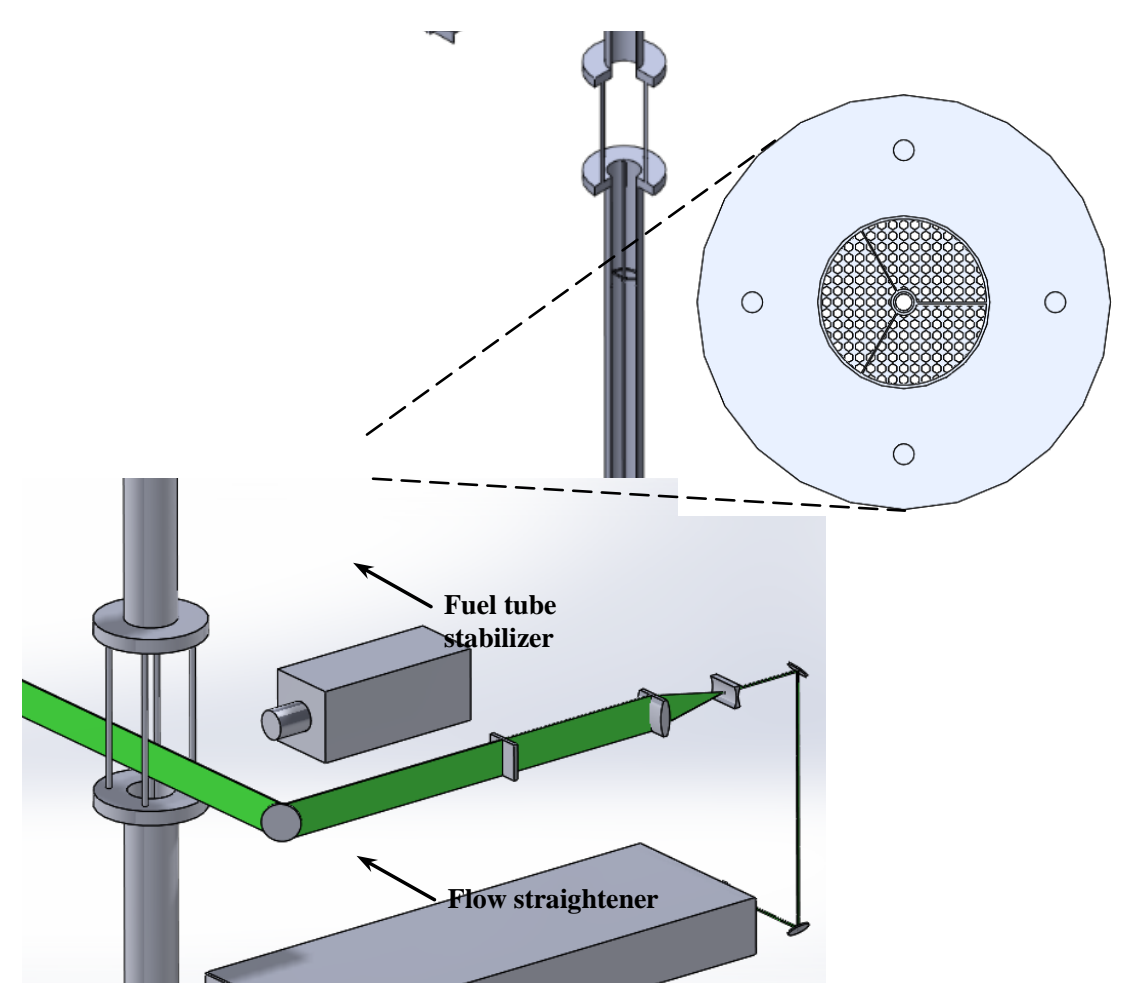

Figure 1 - Picture of the vertical co-flowing non-premixed flame experimental facility (left), a detailed view of the burner (top-right), and a model showing the PIV laser diagnostic setup and the beam path (bottom-right).

It is known that, for low Froude numbers, i.e. the buoyancy dominated regime, non-premixed flames are globally unstable and exhibit narrowband oscillations at a low frequency on the order of $f_{b} \sim 10 \mathrm{~Hz}$ $[32,33]$. When present in this facility, these instabilities were quite evident from the presence of a low frequency peak in the chemiluminescence images. All data presented in this paper were obtained from conditions where this peak was at least 20 times smaller than the spectral peak at the forcing frequency, over a large axial extent. Figure 2 shows representative spectra of the flame position, $\xi$, for an unforced and forced system. Note the absence of the flicker frequency, and the dominance of the forcing frequency at $f_{f}$ for the forced system. The burner tube diameter was chosen as large as possible, with the constraint of having a ratio of $R_{f} / R_{w} \ll<1$ (to minimize confinement effects) and a momentum-dominated flame. The flow velocities were set by balancing between the competing requirements of a laminar flow and a momentum dominated flame. For reference, a flow velocity of $40 \mathrm{~cm} / \mathrm{s}$ corresponds to Reynolds numbers on the order of $\sim 200$ and 2000 , based upon fuel and oxidizer port diameters, respectively. 

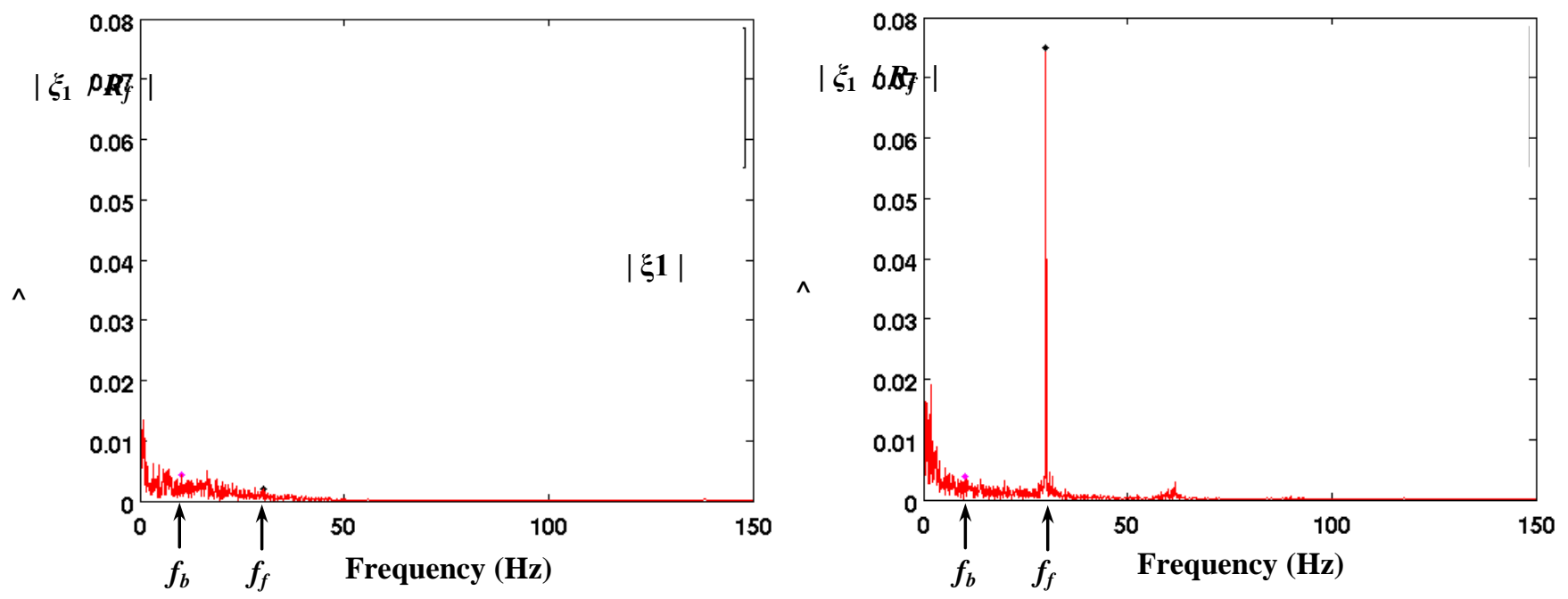

Figure 2 - Frequency spectra of the transverse flame location, $\xi_{1}$, at $\mathrm{x} / R_{f}=5.13$ for the $U_{0}=0.4 \mathrm{~m} / \mathrm{s}$ case for the unforced (left) and forced at $30 \mathrm{~Hz}$ (right) configurations. Vertical arrows indicate the forcing frequency, $f_{f}$, and the buoyant instability frequency, $f_{b}$.

Moving into the combustor section, the fuel and air tubes terminate into a $30.5 \mathrm{~cm}$ long cylindrical quartz tube of inner radius $R_{w}=3.75 \mathrm{~cm}$ and $2.0 \mathrm{~mm}$ wall thickness, that provides confinement and minimizes the influence of ambient disturbances on the flame, whilst allowing optical access from all sides. The combustion products then flow into an exhaust section, which consists of a $0.6 m$ long tube of inner radius $R_{w}=4.0 \mathrm{~cm}$ and $5.0 \mathrm{~mm}$ wall thickness. Connected perpendicular to this exhaust, $0.46 \mathrm{~m}$ above the termination of the combustor section, are two antipodal $30.5 \mathrm{~cm}$ long tubes of inner radius $1.75 \mathrm{~cm}$ and $3.55 \mathrm{~mm}$ wall thickness, which are attached to loudspeakers. These loudspeakers are driven by a harmonic function generator and amplifier, in order to obtain desired forcing amplitudes, i.e. $\varepsilon=\hat{u}_{1} / U_{x, 0}$ values, between $0.05-0.3$. In general, this forcing configuration excites both axial (i.e., onedimensional) acoustic disturbances and transverse, multidimensional duct modes. However, the 10$100 \mathrm{~Hz}$ forcing frequency range used for this study is well below the $\sim 4930 \mathrm{~Hz}$ cut-off frequency, defined by $f_{c}=0.58 \mathrm{c} / R_{w}$, of the exhaust section [5]. Consequently, multi-dimensional disturbances are evanescent and decay quickly in the axial direction. As such, the acoustic excitation source utilized here 
is essentially one-dimensional. For reference, the first transverse duct mode decays as $\exp \left[-\sqrt{m^{2} \pi^{2}-\left(k R_{w}\right)^{2}} \frac{x}{R_{w}}\right][5]$, where $k=\sqrt{\omega^{2} / c^{2}}$, so that its value is less than $1.4 \times 10^{-6} \%$ of its original value once it reaches the combustor section.

The primary experimental diagnostics consisted of high speed, line of sight integrated, imaging of flame luminosity, and high speed particle image velocimetry (PIV), see Figure 1. High speed imaging was performed with a Photron SA5 camera, operating at a $1000 \mathrm{~Hz}$ frame rate, with $0.2 \mathrm{~ms}$ exposure time, and $1024 \times 1024$ pixel resolution. The camera was outfitted with a f/2.8, $135 \mathrm{~mm}$ lens, such that a physical window of $90 \mathrm{~mm} \times 90 \mathrm{~mm}$ region of the test section was imaged. This imaging region spanned from the burner lip to $15 R_{f}$ downstream. The flame was imaged through a bandpass optical filter, centered at $527 \mathrm{~nm}$, with greater than $93 \%$ transmission between $517 \mathrm{~nm}$ and $537 \mathrm{~nm}$. A total of 10,918 images were acquired in each run. Figure 3 shows two representative luminosity images of the forced non-premixed flame, along with an image of a forced premixed flame for comparison. Notice the clear presence of wrinkles along the flame surface. However, the premixed flame develops a prominent cusp downstream, a manifestation of flame propagation, which is absent in non-premixed flames.
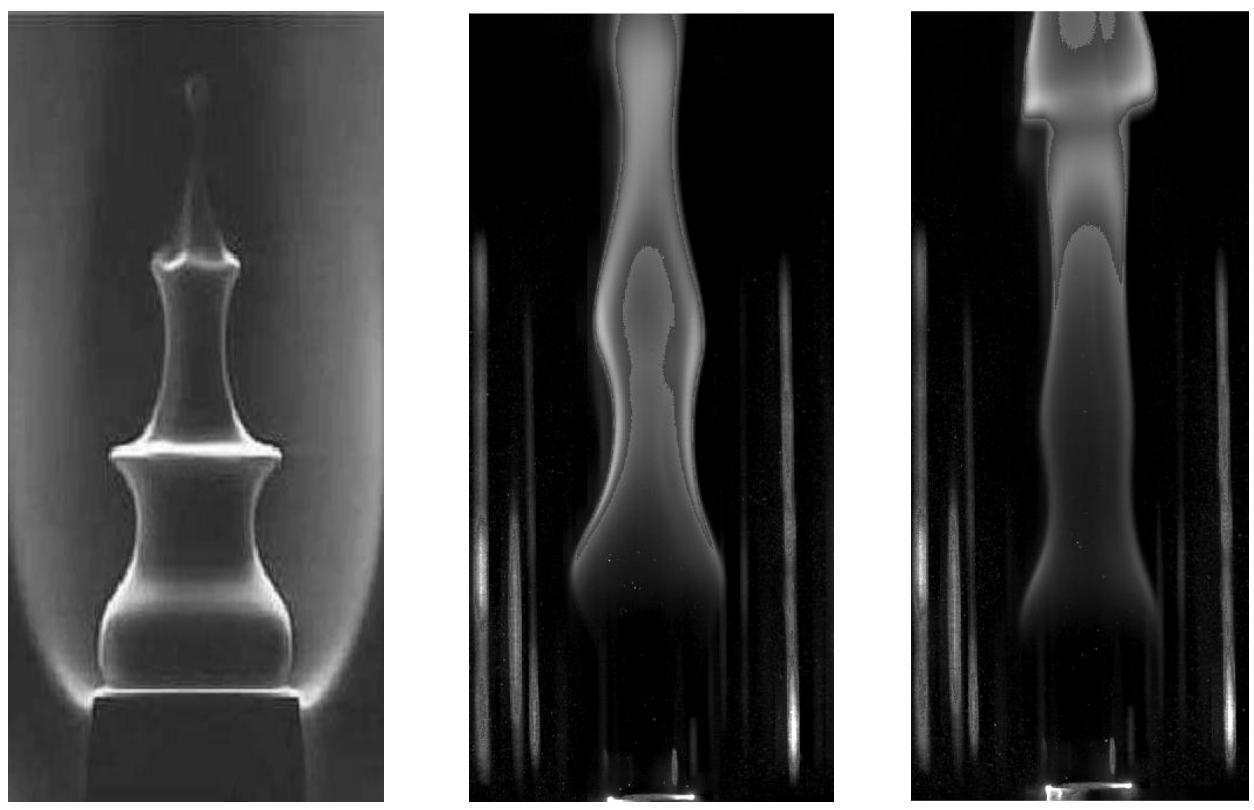
Figure 3 - Schlieren image of a conical methane-air forced premixed flame at $150 \mathrm{~Hz}$ (left) (reproduced from Ducruix et al. [34]) along with line of sight luminosity images from the present study of forced methane-air nonpremixed flames at $30 \mathrm{~Hz}$ (middle) and $50 \mathrm{~Hz}$ (right) forcing frequency.

Mie scattering images for high speed PIV were also obtained. A dual-flow seeder was utilized to independently seed both the fuel and air flows with $0.3-1 \mu m \mathrm{Al}_{2} \mathrm{O}_{3}$ particles, chosen due to their durability in reacting flows and their high refractive index [35]. This particle size was chosen due to the relatively low flow velocities used in this testing, so that the particles could accurately track the flow at the frequencies of interest. For reference, the Stokes number, representing a characteristic nondimensional frequency of the particle response, for the largest excitation frequency considered $(100 \mathrm{~Hz})$ is $S k=0.004$, from the definition $S k=d_{p} \sqrt{\omega / v}$ [35], utilizing a nominal particle size of $0.7 \mu m$ and a viscosity of $v=1.72 \times 10^{-5} \mathrm{~m}^{2} / \mathrm{s}$, calculated at $298 \mathrm{~K}$. The Stokes number is 4 times smaller, $S k=0.001$ when calculated at the approximate product temperature, $1800 \mathrm{~K}$. The seed was dried in an oven for an excess of 24 hours, then placed into the two separate vertical seed holders. During operation, the fuel and air flows passed thru supercritical orifices, directly below the seed holders, before passing through the seed. A pneumatic shaker was attached adjacent to both seed holders, to ensure continued seed pickup.

The particle-laden flow then passed through the respective passages to the combustor, where they were illuminated with a double-pulsed Nd:YAG laser, with wavelength of $532 \mathrm{~nm}$, pulse duration of $100 \mathrm{~ns}$, and $4 \mathrm{~mJ} /$ pulse energy. Each of the two lasers in the double-pulsed system is operated at $500 \mathrm{~Hz}$. The laser beams were expanded with a $-50 \mathrm{~mm}$ cylindrical lens into an expanding sheet, which was then shaped with a $750 \mathrm{~mm}$ cylindrical lens. The time-delay between the two illuminating laser pulses of a given pair was $1 \mathrm{~ms}$, chosen such that for our intended flow velocity range, seed particles would move roughly $1 / 4^{\text {th }}$ of the PIV (final pass) interrogation window (to be discussed next). This is a significant enough spatial change to ensure good velocity calculations, while reducing loss of pairs. The 
measurement plane was located through the concentric central axis of the fuel and oxidizer ports, as shown by Figure 3. Defocusing introduces challenges due to the high curvature of the non-premixed flame sheet as will be discussed later in the experimental results.

Data was obtained over a parametric grid of experimental input variables, specifically flow velocity, forcing frequency, and forcing amplitude, all of which have been shown to be dynamically significant factors for forced flames. These conditions are summarized in Table I, where $\varepsilon$ is the forcing amplitude parameter, defined as $\varepsilon=\left|\hat{u}_{x, 1}\left(\odot, f=f_{f}\right)\right| / U_{x, 0}(\odot)$, evaluated at a reference coordinate, $\odot$, located well into the oxidizer stream.

Table I - Range of experimental parameters.

\begin{tabular}{|l|l|}
\hline \multicolumn{1}{|c|}{ Input parameter } & \multicolumn{1}{c|}{ Values } \\
\hline Fuel/Air flow velocity, $U_{0, \mathrm{des}}(\mathrm{m} / \mathrm{s})$ & $0.1,0.2,0.4,0.7$ \\
\hline Forcing Frequency, $f_{f}(\mathrm{~Hz})$ & $10,20,30,40,50,70,100$ \\
\hline Forcing Amplitude, $\varepsilon$ & $0.05,0.15$ \\
\hline
\end{tabular}

\section{Data Analysis}

The two key experimental observables are the instantaneous flame position, and instantaneous velocity field at the flame. The processing methods for extracting these data are described in this section. A representative luminosity/Mie image of a wrinkled non-premixed flame, obtained from our experimental setup, is shown in Figure 4(a). These images are used to characterize the space-time dynamics of the flame sheet, which requires determining the instantaneous flame edge. 


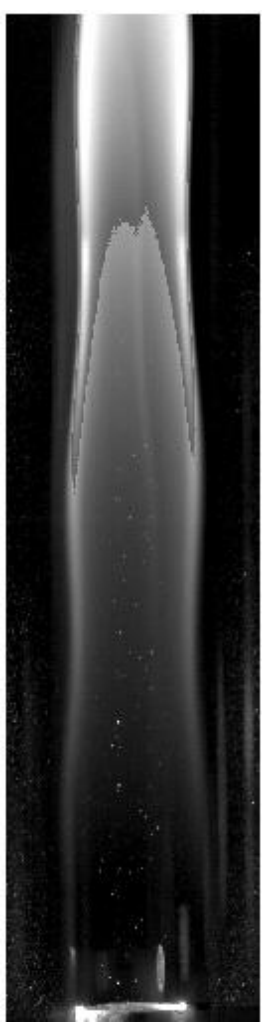

(a)

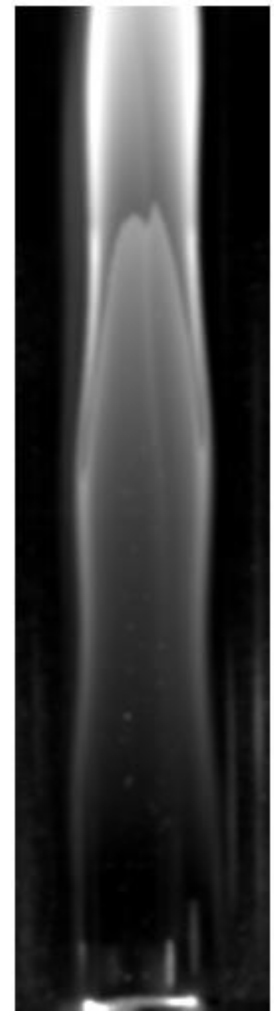

(b)

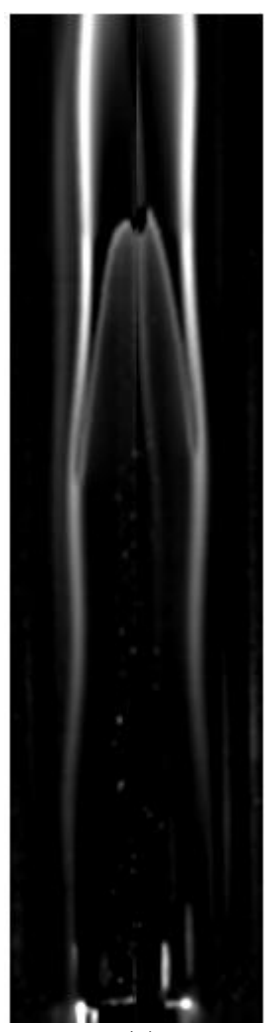

(c)

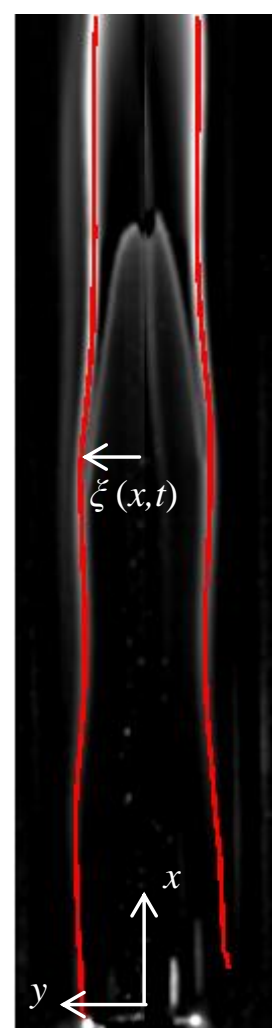

(d)

Figure 4 - (a) Raw luminosity image of a forced non-premixed flame (b) bilateral filtered image (c) inverse Abel transformed image (d) and edge tracked image.

To reduce noise without blurring edges, a bilateral filter was used. Implementing this spatially varying nonlinear filter, the intensity value at each spatial location was replaced by a weighted average of the intensity values from nearby locations. The weights, based on a Gaussian distribution, are dependent upon both spatial orientation as well as quantitative intensity differences. Systematically looping through each location and modifying the weights accordingly makes this procedure edge preserving, as can be seen from Figure 4(b). An inverse Abel transform was then used on the left and right halves of the images, independently (being a symmetric operator), in order to estimate an intensity slice from the line-of-sight integrated image. The combination of these procedures is shown in Figure 4(c). The flame edge, defined as the instantaneous transverse location of the calculated edge and denoted $\xi(x, t)$, was then determined from the intensity peak, shown in Figure 4(d). Note that an edge is calculated separately for the left and right transformed images - these two values would coincide if the images 
were perfectly axisymmetric, and for our results they generally agree to within 5\% (comparisons are shown later; see Figure 12). The Fourier transform of the fluctuating component $\xi_{1}(x, t)$, denoted $\hat{\xi}_{1}(x)$, then quantifies the wrinkle amplitude and phase (with respect to its value at the left flame branch base) as a function of axial location.

We next describe the velocity calculations, obtained from the PIV measurements. Velocity vectors were computed from the Mie scattering image pairs using a cross-correlation algorithm [36] with a multi-pass approach [37] and two-dimensional Gaussian sub-pixel interpolation [36]. Image preprocessing was implemented with a particle intensity normalization of 3 pixels. The multi-pass analysis consisted of two preliminary passes with $64 \times 64$ pixel interrogation windows and $75 \%$ overlap between interrogation windows, followed by 2 passes with $24 \times 24$ pixel interrogation windows and $50 \%$ overlap between the interrogation windows, resulting in velocity vectors calculated at $0.8 \mathrm{~mm} \times$ $0.8 \mathrm{~mm}$ spatial intervals. Post-processing algorithms were used to discard a small number of spurious vectors, defined as points with 1.7 times the root mean square (rms) values of the neighbors. In addition, a local validation method discarded neighboring vectors whose ratio of difference-to-average velocity magnitude was greater than 1.9 times the rms of the nearest neighbors. These post-processing techniques resulted in less than $1 \%$ of the total velocity vectors being discarded. Discarded values were replaced with the instantaneous average of neighboring vectors.

The PIV measurement was calibrated by imaging a black anodized aluminum calibration plate with a laser etched pattern, which was located in-plane with the laser light sheet. The laser etched pattern consisted of a regularly spaced grid of crosses, composed of $0.3 \mathrm{~mm}$ thick lines, with spacing of $6 \mathrm{~mm}$ and with positional uncertainty of $0.004 \%$. The spatial coordinates of the Mie scattering images and resultant velocity fields were mapped to this grid. The worst-case calibration error was estimated to be 1 pixel over the large $160 \mathrm{~mm}$ calibration plate. 
Near the flame tip, the highly curved flame defocuses the cameras view of the Mie particles, sometimes blurring them. When compounded with the luminosity emissions that partially saturate the signal at flame tip, these effects lead to highly spurious results. An example image displaying this effect is shown in Figure 5, showing the spurious velocity data near the upper portion of the image.

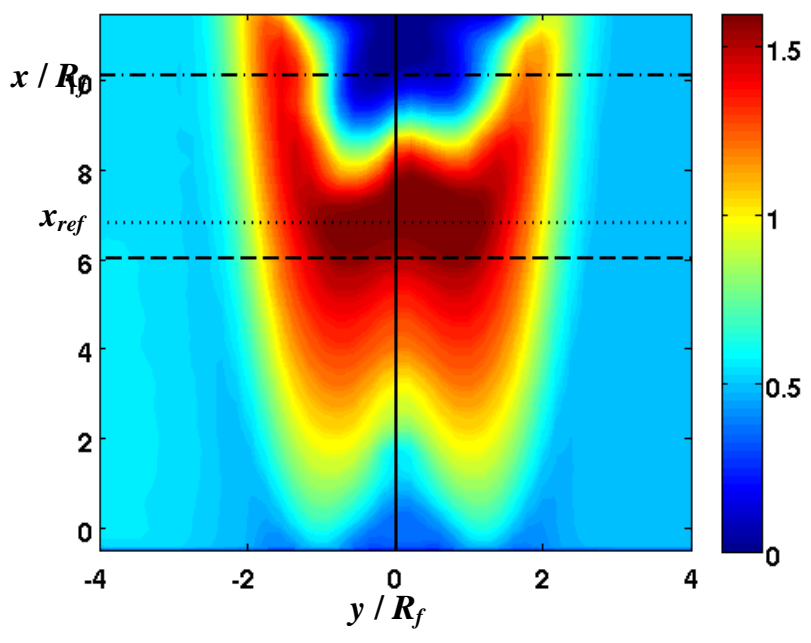

(a)

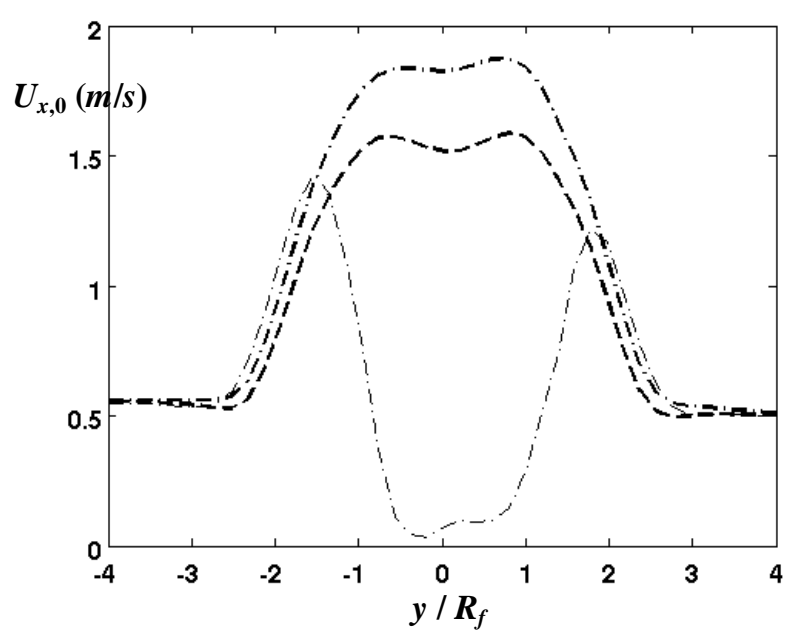

(c)

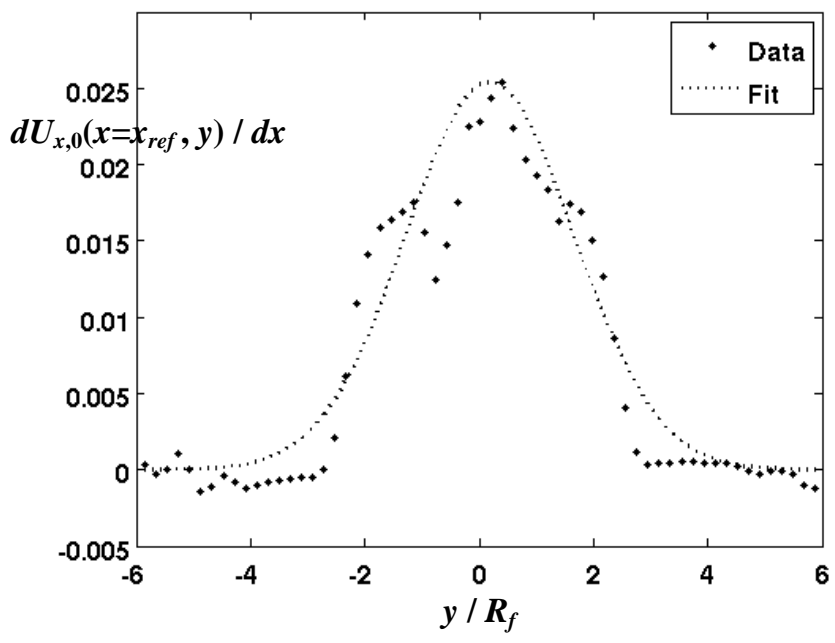

(b)

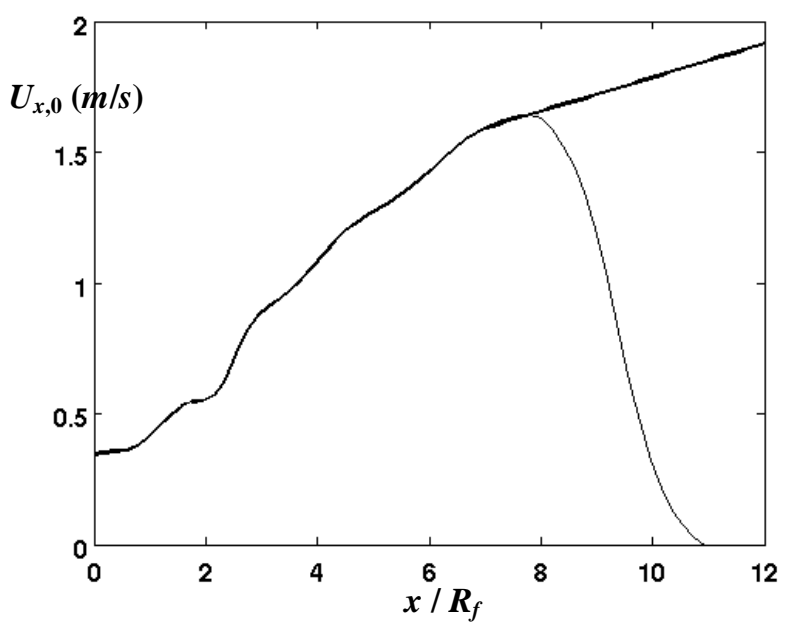

(d)

Figure 5 - Representative PIV data from Case C (listed in Table II) showing spurious result near upper edge of image due to intensity saturation and flame curvature distortion (a). The void filling procedure is also depicted by the axial velocity gradient, $\mathrm{d} U_{x, 0} / \mathrm{dx}$ at a representative axial location below the void, $x_{r e f}(\mathrm{~b})$, along with transverse (c) and axial (d) cuts of the raw velocity data (thin lines) and extrapolated data (thick lines). The line styles correspond to the various traverses show in top left image. 
In these regions, the velocity is extrapolated from upstream locations of good data. This is done by looking at the axial velocity gradient, i.e. $d U_{x, 0} / d x$ at an axial location upstream (below the void), where valid data exists, denoted $x_{r e f}$. A Gaussian curve is fit to this gradient profile, shown in Figure 5, and the downstream velocity values are then extrapolated at locations $x>x_{\text {ref. }}$ Representative transverse and axial cuts are also shown in Figure 5, showing both the raw and extrapolated data. The calculation results shown later will indicate the regions of actual and extrapolated data, by denoting $x_{r e f}$.

\section{Experimental Results}

Having the ability to track the instantaneous flame edge, characteristics of the experimental flame wrinkle dynamics can be investigated. We will refer to four representative conditions throughout the rest of this paper, shown in Table II.

Table II -Data sets used in Experimental Results section

\begin{tabular}{|l|l|l|l|l|}
\hline \multicolumn{1}{|c|}{ Case Set } & \multicolumn{1}{c|}{$\boldsymbol{U}_{\mathbf{0}, \text { des }}(\boldsymbol{m} / \mathbf{s})$} & $\boldsymbol{f}_{\boldsymbol{f}}(\boldsymbol{H z})$ & \multicolumn{1}{c|}{$\boldsymbol{E}$} \\
\hline Case A & 0.1 & 50 & 0.15 \\
\hline Case B & 0.2 & 20 & 0.05 \\
\hline Case C & 0.4 & 30 & 0.05 \\
\hline Case D & 0.4 & 40 & 0.05 \\
\hline
\end{tabular}

Representative results showing the instantaneous spatial dependence of $\xi(x, t)$ at different time instances over a forcing period is shown in Figure 6. Notice the clear downstream axial convection of the flame wrinkle crests with time, a result predicted by Eq.(1). Flame wrinkle convection can also be directly inferred from the phase of $\hat{\xi}\left(x, f=f_{f}\right)$. Note that a wrinkle convecting axially at a constant speed, $U_{c}$, leads to a linear phase-axial location dependence, with a slope given by: 


$$
U_{c}=\left|\frac{2 \pi f_{f}}{\partial \angle \hat{\xi}\left(x, f=f_{f}\right) / \partial x}\right|
$$

Figure 6 also plots the axial dependence of $U_{c}$ calculated using this formula, overlaid upon measured steady velocity results along various three axial profiles through the domain. Note the very close correspondence of the wrinkle velocity to the mean flow velocity. This result is consistent with prior analytical work that used simpler model velocity profiles, and showed that to leading order in $P e, U_{\mathrm{c}}=$ $U_{x, 0}$. Note that this is not the case in premixed flames, where the flame wrinkle speed is the vector superposition of the flow velocity and flame speed projected normal to the flame front.
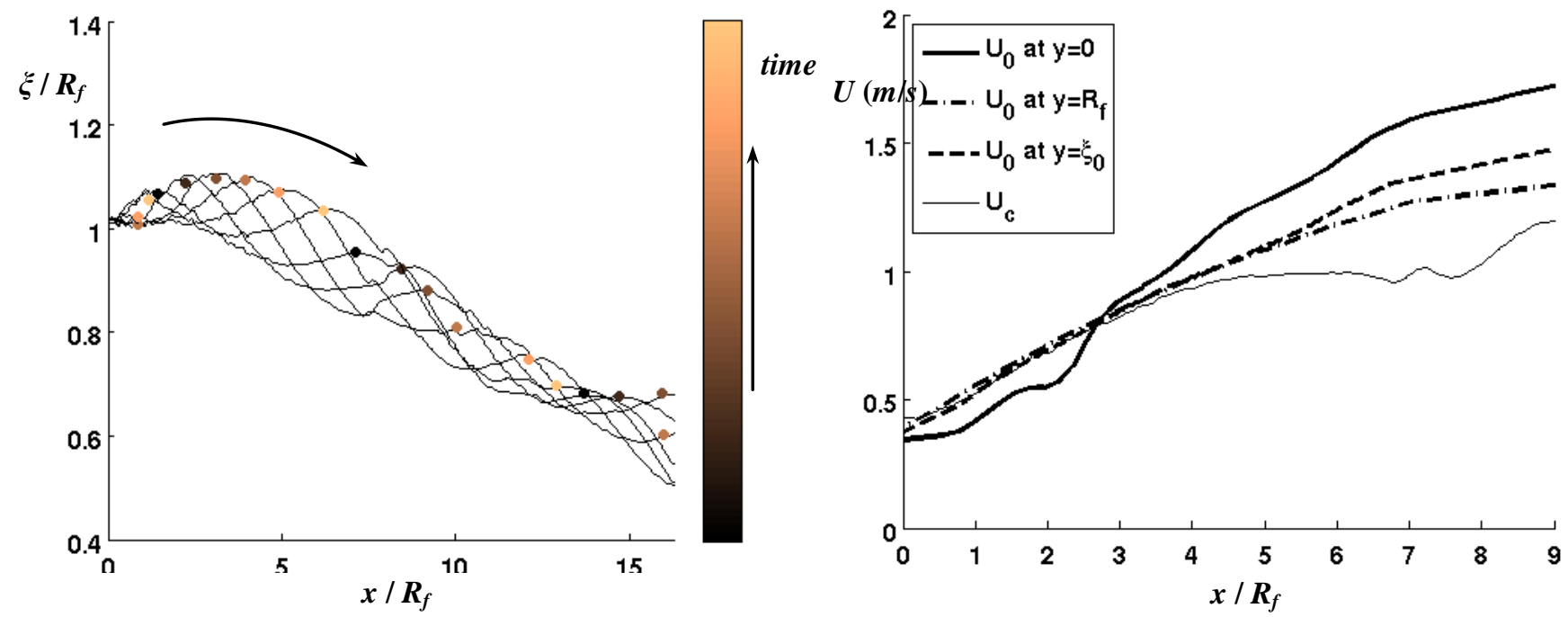

Figure 6 -Instantaneous flame positions at various time instances over a forcing period (left; experimental data, where arrows indicate increasing time) and steady flow velocity cuts, at the centerline $[y=0]$, burner lip $\left[y=R_{f}\right]$, and mean flame position $\left[y=\xi_{0}(x)\right]$, and wrinkle convection speed (right) results for Case $\mathrm{C}$.

The fluctuating flame position, $\xi_{1}(x, t)$ is calculated as the transverse distance of the instantaneous flame position from the average; i.e., $\xi_{1}(x, t)=\xi(x, t)-\xi_{0}(x)$. The fluctuating response can then be extracted from the Fourier transform at the forcing frequency, $\hat{\xi}\left(x, f=f_{f}\right)$. Representative results for the magnitude and phase of the flame wrinkle are shown in Figure 7 for Case C. 

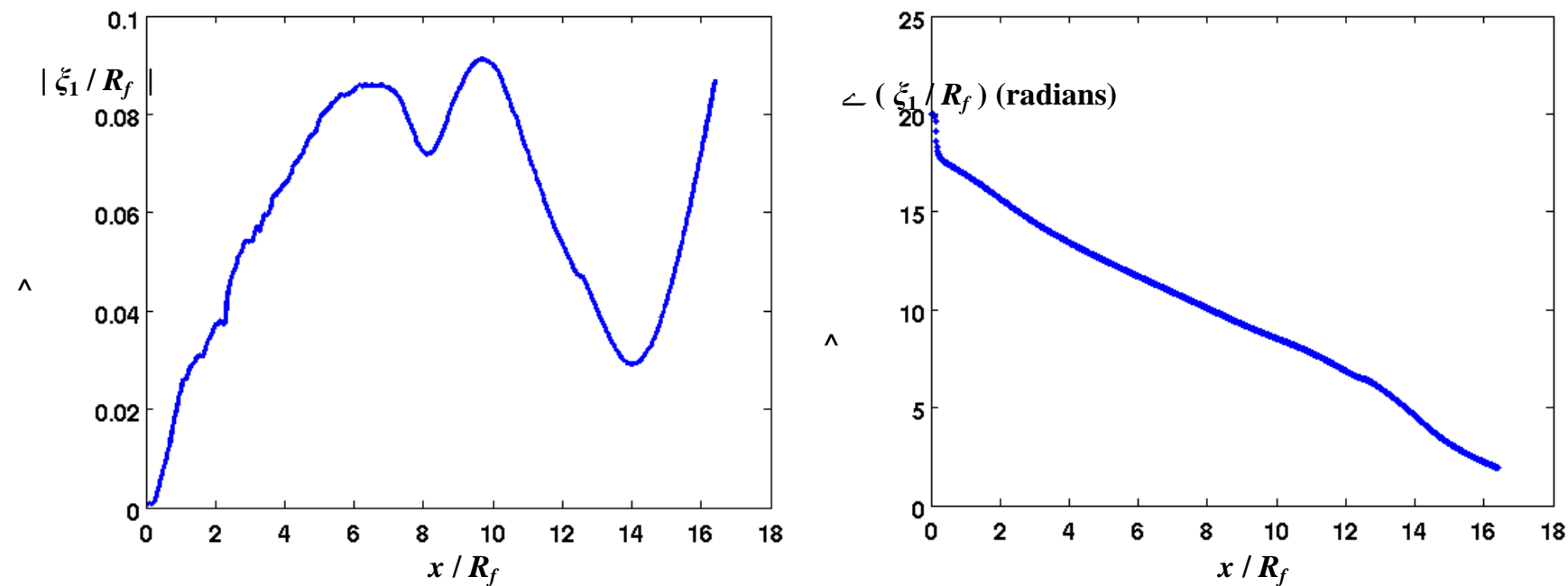

Figure 7 - Representative experimental flame wrinkle amplitude (left) and phase (right) results for Case C.

Note the nearly stationary behavior of the flame at the base near the burner lip, i.e. $\hat{\xi}_{1}\left(x \sim 0, f=f_{f}\right) \sim 0$, indicative of flame attachment - these results are consistent with prior theoretical results developed for non-premixed flames in the fast chemistry, $P e>>1$ limit [38]. Additionally in this near base region, the response magnitude grows with downstream distance. Further downstream the magnitude of flame wrinkling peaks at $x / R_{f}=6$ and oscillates. This behavior is suggestive of an interference phenomenon and is consistent with the predictions of prior calculations of forced nonpremixed flames, such as Eq.(1). Similar interference results have been observed for axially forced premixed flame systems [39]. The phase rolls off nearly linearly with axial distance, for the reasons discussed earlier in the context of Figure 6. Additional flame wrinkle results will be shown in Section 6 where we compare measured results with analysis.

We next show representative velocity results. The time averaged axial $U_{x, 0}$ and radial $U_{r, 0}$ velocity component fields are shown in Figure 8 for the same conditions shown in Figure 5 thru Figure 7 . The time averaged centerline of the flame, extracted from the procedure outlined in Figure 4 and demonstrated instantaneously in Figure 6, is also indicated. The figure clearly shows the axial flow acceleration. In addition, the radial velocity result shows the induced radially expanding (both inflowing 
and outflowing) velocities inside and outside the flame, respectively, an expected result based on the gas expansion occurring on both sides of the flame sheet.
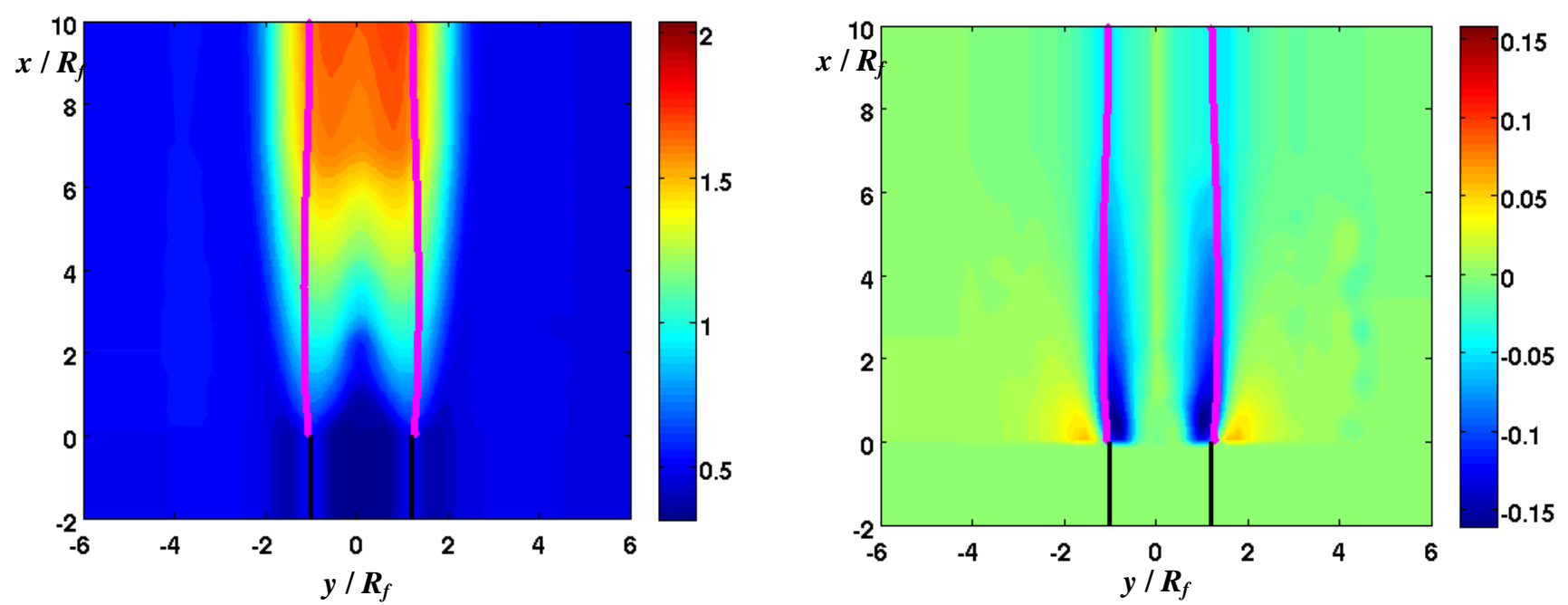

Figure 8 - Representative experimental velocity fields (using corrections described in the context of Figure 5) showing the time averaged axial, $\mathrm{U}_{x, 0}$ (left), and radial, $\mathrm{U}_{r, 0}$ (right; positive direction being radially outward), velocity fields for Case $\mathrm{C}$, along with overlaid steady flame position (line). Fuel port walls are shown in black and the colorbars show velocity in $\mathrm{m} / \mathrm{s}$.

Figure 9 shows the axial and radial magnitude and phase values of the velocity fluctuations at $\xi_{0}(x)$ for Case C. The phase of the radial velocity disturbance shows clear signs of downstream convection (the phase slope corresponds to a value of $\sim 0.6 \mathrm{~m} / \mathrm{s}$ ), suggesting that it is dominated by vortical disturbances. Being convectively unstable in its own right, harmonic excitation causes the shear layer to roll up into vortices at the frequency of excitation. The axial velocity phase is more complex, but its general flatness with respect to axial distance is more suggestive of it being dominated by an acoustic standing wave. Also, the complex axial disturbance magnitude distribution is perhaps due to the influence of the flame density jump upon the acoustic field. 

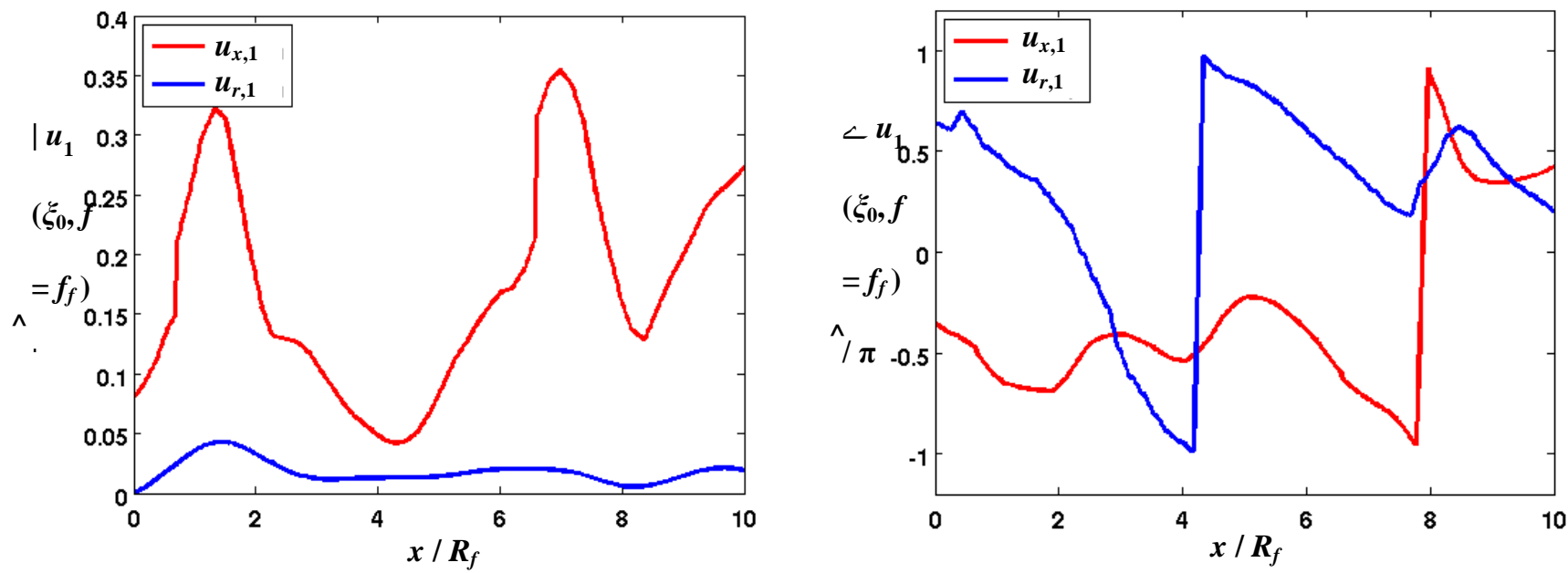

Figure 9 - Representative experimental axial, $\hat{u}_{x, 1}$, and radial, $\hat{u}_{r, 1}$, disturbance velocity magnitude (left) and phase (right) curves extracted along the mean flame for Case $\mathrm{C}$.

\section{Flame Position Modeling}

This section presents the flame dynamics modeling approach, which utilizes the experimentally measured mean and fluctuating velocity fields as inputs to the mixture fraction equation. The predicted space-time dynamics of the reaction sheet are extracted from these calculations and compared to the actual measured flame positions for various flow velocities and forcing frequencies.

\subsection{Equation Formulation}

This section presents the background equations and corresponding assumptions fundamental to this analysis. The key assumptions of this analysis are (1) infinitely fast chemistry, wherein the flame sheet collapses to the stoichiometric surface of the mixture fraction field, $\boldsymbol{Z}_{s t},(2)$ all species have equal diffusivities, (3) negligible radiative heat loss effects, (4) small perturbation amplitudes so that the products of disturbances can be neglected, (5) unity Lewis number, and (6) local diffusivity is only a function of temperature and determined from the Hirschfelder-Bird-Spotz equation [40]. 
Following assumption (2), the species conservations can be manipulated using standard methods to develop the mixture fraction equation, referred to here as the $z$-equation [41]:

$$
\frac{\partial Z}{\partial t}+\vec{u} \cdot \nabla Z=\nabla \cdot(\mathscr{Q} \nabla Z)
$$

The velocity and diffusion coefficient terms are completely general and vary in space and time. Note that prior analytical treatments of this problem have prescribed these profiles, generally using relatively simple spatial profiles to enable analytical tractability. As this equation is solved computationally in this study, no such assumption is necessary.

Following assumption (1), the flame position coincides with the surface $Z(\vec{x}, t)=Z_{s t}=1 /\left(1+\varphi_{o x}\right)$. The instantaneous position of this reaction sheet is defined by $y=\xi(x, t)$, as shown in Figure 4 . Following assumption (4), each variable is expanded as ()$(x, t)=()_{0}(x, y)+()_{1}(x, y, t)$. Working in cylindrical coordinates, the resulting steady and fluctuating mixture fraction field governing equations are:

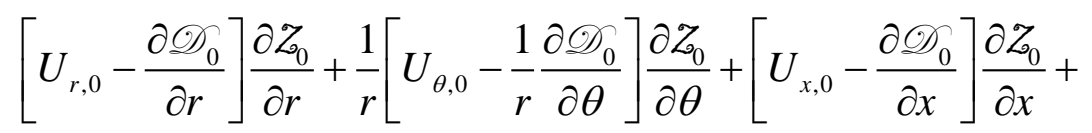

$$
\begin{aligned}
& -\mathscr{Q}_{0}\left[\frac{1}{r} \frac{\partial \mathscr{Z}_{0}}{\partial r}+\frac{\partial^{2} Z_{0}}{\partial r^{2}}+\frac{1}{r^{2}} \frac{\partial^{2} Z_{0}}{\partial \theta^{2}}+\frac{\partial^{2} Z_{0}}{\partial x^{2}}\right]=0 \\
& \frac{\partial \mathscr{Z}_{1}}{\partial t}+\left[U_{r, 0}-\frac{\partial \mathscr{\mathscr { V }}_{0}}{\partial r}\right] \frac{\partial \mathscr{Z}_{1}}{\partial r}+\left[\frac{U_{\theta, 0}}{r}-\frac{1}{r^{2}} \frac{\partial \mathscr{\mathscr { V }}_{0}}{\partial \theta}\right] \frac{\partial \mathscr{Z}_{1}}{\partial \theta}+\left[U_{x, 0}-\frac{\partial \mathscr{\mathscr { V }}_{0}}{\partial x}\right] \frac{\partial \mathscr{Z}_{1}}{\partial x}+ \\
& -\mathscr{Q}_{0}\left[\frac{1}{r} \frac{\partial Z_{1}}{\partial r}+\frac{\partial^{2} Z_{1}}{\partial r^{2}}+\frac{1}{r^{2}} \frac{\partial^{2} Z_{1}}{\partial \theta^{2}}+\frac{\partial^{2} Z_{1}}{\partial x^{2}}\right]=\left[-u_{r, 1}+\frac{\partial \mathscr{O}_{1}}{\partial r}\right] \frac{\partial Z_{0}}{\partial r}+\left[-\frac{u_{\theta, 1}}{r}+\frac{1}{r^{2}} \frac{\partial \mathscr{Q}_{1}}{\partial \theta}\right] \frac{\partial Z_{0}}{\partial \theta}+
\end{aligned}
$$

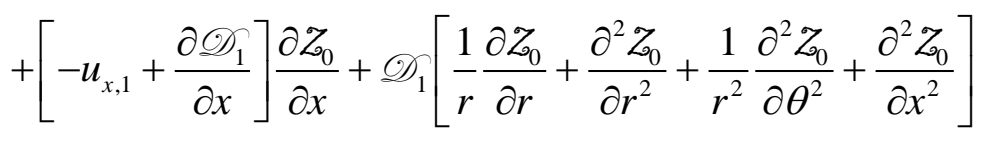


Thus, provided adequate inputs, such as prescribed diffusion coefficient and velocity fields over the entire computational domain, along with boundary conditions, the steady and fluctuating mixture fraction fields can be solved for over the domain of interest.

Part of these necessary inputs, i.e. the prescribed steady and fluctuating velocity fields, were experimentally obtained and presented in Section 4, while others, such as the boundary conditions, will be discussed in the next subsection. The sole remaining quantity of concern is the spatially variant diffusion coefficient, which can be re-written using the chain rule as:

$$
\frac{\partial \mathscr{\partial}}{\partial x_{i}}=\frac{\partial \mathscr{D}}{\partial T} \frac{\partial T}{\partial Z} \frac{\partial Z}{\partial x_{i}}
$$

The specification of $\partial \mathscr{O} / \partial T$, following assumption (6), was calculated via the Hirschfelder-BirdSpotz equation, with empirical correlation coefficients developed by Fuller specifically for methane air systems as [42]:

$$
\mathcal{Q}_{\mathrm{CH}_{4}-\mathrm{Air}}\left[\mathrm{cm}^{2} / \mathrm{s}\right]=\frac{10^{-3} T^{1.75}\left(\frac{1}{M_{\mathrm{CH}_{4}}}+\frac{1}{M_{A i r}}\right)^{1 / 2}}{P\left[\left(V_{\mathrm{CH}_{4}}\right)^{1 / 3}+\left(V_{\mathrm{Air}}\right)^{1 / 3}\right]^{2}}
$$

where $P, M$, and $V$ are the absolute pressure in atmospheres, molecular weights, and atomic diffusion volumes [43] of the various species, i.e. methane and air. The pressure is assumed to be ambient along with the reference values for the molecular weights and diffusion volumes. Similarly, $\partial T / \partial Z$ is determined using assumption (5) of unity Lewis number, where the temperature dependence can be related to the mixture fraction by: 


$$
\begin{array}{ll}
Z<Z_{s t} & T(Z)=\frac{T_{a}-T_{f}}{\left(-Z_{s t}\right)}\left(Z-Z_{s t}\right)+T_{f} \\
Z>Z_{s t} & T(Z)=\frac{T_{a}-T_{f}}{\left(1-Z_{s t}\right)}\left(Z-Z_{s t}\right)+T_{f}
\end{array}
$$

\subsection{Boundary Conditions and Inflow Mixture Fraction Specification}

There are a number of complications in comparing experimental and numerical data for the nonpremixed problem, which are not present in the premixed flame problem. In prior related studies for premixed flame dynamics, all modeling inputs can be directly obtained from measurements - these are the velocity field at the flame (i.e., the mean and fluctuating velocity field upstream over the entire experimental domain is not required, as it is for the non-premixed problem) and the time averaged position of the flame [7]. In contrast, due to the elliptic nature of the mixture fraction equations, it is not sufficient to only specify the time averaged flame position and fluctuating velocity field at the flame. Rather, as shown by Eq.(3), the mixture fraction field, its gradient, and the fluctuating velocity field in the whole domain must be specified. This section describes the boundary conditions and approach used to specify the velocity field in regions outside of the measurement window, as well as the time averaged mixture fraction field, which was not measured.

A physical window of $90 \mathrm{~mm} \times 90 \mathrm{~mm}$ region of the test section was imaged, oriented symmetrically above the fuel port, spanning from the burner lip to $15 R_{f}$ downstream, with PIV velocity data available as far as $12 R_{f}$ downstream. Figure 10 shows these various regions schematically. As the transverse extent of the PIV camera capture region, $\sim 7.5 R_{f}$, was smaller than the total experimental region, $\sim 10 R_{f}$, the velocity field in the exterior radial region, i.e. $r>7.5 R_{f}$, was determined by extrapolation of the measurements in the viewing window. Figure 5 shows how these values become constant far into the oxidizer domain, and thus $U_{0}\left(x, r>R_{f}\right)$ was set equal to $U_{0}\left(x, r=7.5 R_{f}\right)$, for both the axial and radial components, respectively. The radial velocity was also set to zero at the walls, $U_{r, 0}\left(x>0, r=R_{w}\right)=0$. 
Similarly, the velocity field was extrapolated downstream, i.e $x>12 R_{f}$, utilizing the procedure previously described in Section 3 and Figure 5. Finally, the velocity in the fuel and oxidizer inflow regions, $x<0$, were set equal to their value at the inlet plane (the velocity field in this region was used to compute the inflow mixture fraction profile, described next); i.e. $U_{x, 0}(x<0, r)=U_{x, 0}(x=0, r)$ was utilized, with no radial component. No penetration boundary conditions were once again utilized at the fuel and oxidizer port walls, i.e. $U_{r, 0}\left(x<0, r=R_{f}\right)=0$. Figure 10 also shows the various regions of actual (textured) and extrapolated (blank space) velocity data. The same extrapolation procedure was utilized on the corresponding fluctuating velocity fields, which are also used as model inputs.

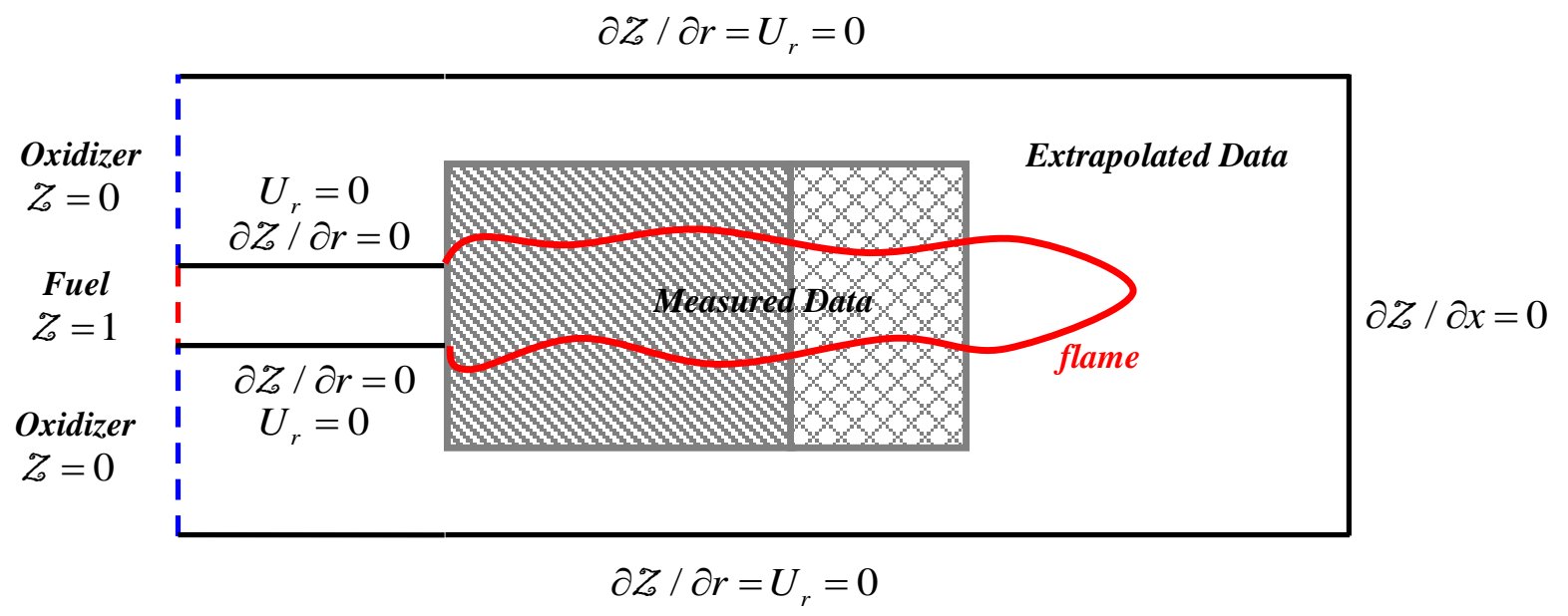

Figure 10 - Schematic of the computational/experimental domain and the regions of various data types, boundary conditions, and inflow conditions. The white, diagonal texture, and cross-hatched textured regions indicate areas of no data, both luminosity and PIV, and only luminosity, respectively.

The mixture fraction inlet profiles, and corresponding field, were determined by assuming pure, i.e. transversely uniform, fuel $(z=1)$ and oxidizer $(z=0)$ reservoirs, also shown in Figure 10, far upstream of the inflow (we used $x=-50 R_{f}$ ). The mixture fraction inflow profile at $x=0$ was not specified, but rather computed as part of the problem, and extracted from the resulting computed mixture fraction field solutions, Eq.(4) and (5). Figure 11 shows various computed time-averaged inflow mixture fraction profiles extracted for a representative $U_{0, \text { des }}$ span. An alternative, more analytically tractable and less 
computationally expensive approach, is to simply assume that at the $x=0$ inlet plane, $Z=1$ for $r \leq R_{f}$ and $z=0$ for $r>R_{f}$. However, axial diffusion of fuel and oxidizer modifies these results near the tube outlet, which was shown to have several dynamically significant effects in a previous study [38]. Consequently, this computational approach was used, which smoothes the mixture fraction profile at $x=0$, an effect which decreases with increasing $U_{0, \text { des }}$; i.e., increasing $P e$ value. Dirichlet boundary conditions were utilized at the upstream reservoir location as, $z=1$ for $r \leq R_{f}$ and $z=0$ for $r>R_{f}$, while Neumann boundary conditions were utilized at the various port and side walls as, $\partial \mathcal{Z} / \partial r=0$, as well as far downstream, $\partial Z / \partial x=0$.

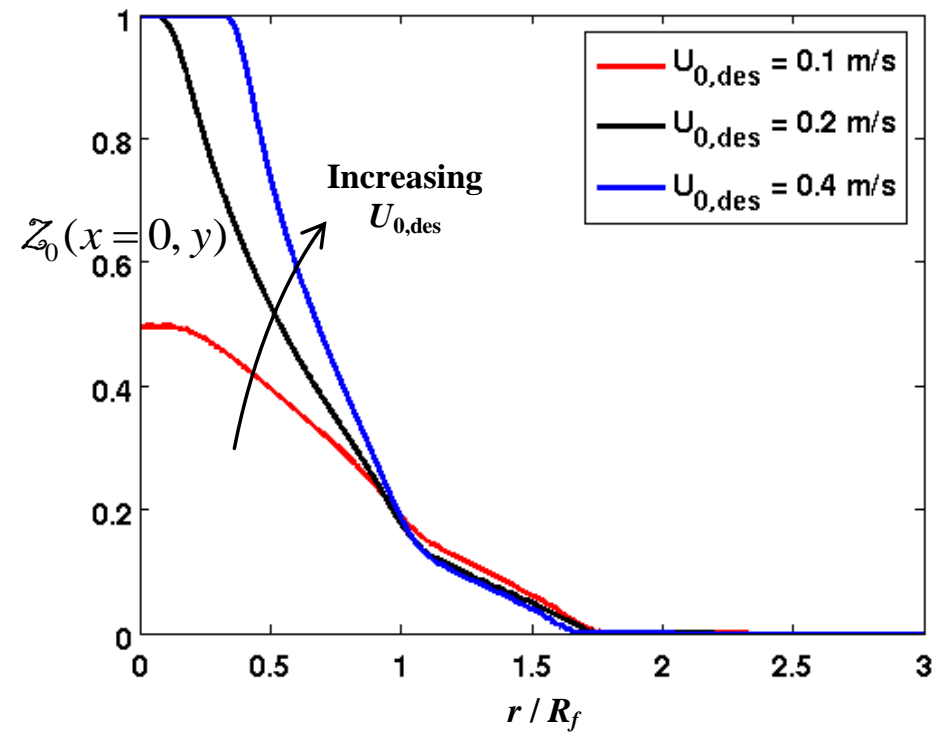

Figure 11 - Computational time-averaged inflow mixture fraction profiles extracted for various experimental cases with $U_{0, \text { des }}$ values of $0.1 \mathrm{~m} / \mathrm{s}, 0.2 \mathrm{~m} / \mathrm{s}$, and $0.4 \mathrm{~m} / \mathrm{s}$.

\subsection{Computational Approach}

Solutions of Eq.(4) and Eq.(5) were obtained with finite element methods, using the Comsol Multiphysics solver due to its balance of computational freedom and efficiency. The multifrontal massively parallel sparse direct solver, denoted MUMPS, was utilized with a convergence criterion set to $10^{-5}$ and relative tolerance of 0.001 . This direct solver is based on LU decomposition and can take 
advantage of all processor cores for increased computational speed. Also, since our previous studies revealed large spatial gradients in the near burner lip region, a mapped rectangular mesh was employed, containing increased grid resolution closer to the fuel port lip. The maximum element size, occurring near the exit plane at $x=18 R_{f}$ was on the order of $R_{f} / 10$, while the minimum element size, occurring near the fuel port lip, was on the order of $R_{f} / 10^{3}$. Grid sensitivity studies were performed which showed that a uniform increase in mesh density by 9 , yielded less than $2 \%$ modification in field and contour results.

As the inflow was experimentally designed and controlled to be non-swirling and laminar, the mean and fluctuating azimuthal velocity was set to zero, resulting in a simplified steady field equation:

$$
\left[U_{r, 0}-\frac{\partial \mathscr{\mathscr { O }}_{0}}{\partial r}\right] \frac{\partial \mathscr{Z}_{0}}{\partial r}+\left[U_{x, 0}-\frac{\partial \mathscr{\mathscr { X }}_{0}}{\partial x}\right] \frac{\partial \mathcal{Z}_{0}}{\partial x}-\mathscr{\mathscr { D }}_{0}\left[\frac{1}{r} \frac{\partial \mathscr{Z}_{0}}{\partial r}+\frac{\partial^{2} Z_{0}}{\partial r^{2}}+\frac{\partial^{2} \mathscr{Z}_{0}}{\partial x^{2}}\right]=0
$$

while the fluctuating equation, Eq.(5), can be cast into spectral space as:

$$
\begin{aligned}
& {\left[U_{r, 0}-\frac{\partial \mathscr{O}_{0}}{\partial r}\right] \frac{\partial \hat{\mathscr{Z}}_{1}}{\partial r}+\left[U_{x, 0}-\frac{\partial \mathscr{\mathscr { D }}_{0}}{\partial x}\right] \frac{\partial \hat{\mathscr{Z}}_{1}}{\partial x}-\mathscr{\mathscr { D }}_{0}\left[\frac{1}{r} \frac{\partial \hat{\mathcal{Z}}_{1}}{\partial r}+\frac{\partial^{2} \hat{\mathcal{Z}}_{1}}{\partial r^{2}}+\frac{\partial^{2} \hat{\mathcal{Z}}_{1}}{\partial x^{2}}\right]=} \\
& =i \omega \hat{Z}_{1}+\left[-u_{r, 1}+\frac{\partial \mathscr{O}_{1}}{\partial r}\right] \frac{\partial \mathscr{Z}_{0}}{\partial r}+\left[-u_{x, 1}+\frac{\partial \mathscr{O}_{1}}{\partial x}\right] \frac{\partial \mathscr{Z}_{0}}{\partial x}+\mathscr{O}_{1}\left[\frac{1}{r} \frac{\partial \mathscr{Z}_{0}}{\partial r}+\frac{\partial^{2} Z_{0}}{\partial r^{2}}+\frac{\partial^{2} Z_{0}}{\partial x^{2}}\right]
\end{aligned}
$$

\subsection{Time-averaged flame shapes and model comparisons}

Once Eq.(9) and (10) are solved, iso- $z$ contours can be extracted from the resulting steady and forced field solutions which, following assumption (1), are utilized to denote the flame sheet position. Figure 12 shows representative results of the $Z_{0}$ fields, where the time averaged flame position is also indicated. If the $Z_{0}$ field were calculated perfectly and the assumptions of this analysis were perfect, the measured flame shape would lie on the $z_{0}=Z_{s t}=0.055$ contour. Clearly it does not - rather, in the 
mid and far-field, i.e. $x / R_{f}>3$, the flame more closely follows the $Z_{0}=Z^{*}=0.11$ contour, while near the burner lip it follows a lower valued iso-contour, dependent upon the specific case. Note how the case on the left shows much better near-field agreement with the experimental results than the case on the right. This difference in near field characteristics is likely a result of errors in the computed $Z_{0}(x=0, r)$ inflow profile. Additionally, the nature of the mixture fraction boundary condition contributes to this discrepancy, requiring the computed flame sheet to attach normally to the fuel port wall, thru use of the no penetration mixture fraction condition, i.e. $\partial Z\left(x<0, r=R_{f}\right) / \partial r=0$ and the assumption of infinitely fast chemistry. In reality, the burner lip has finite thickness and attachment occurs at some finite angle, and the flame will originate some small distance from the wall hence lengthening the steady flame shape, and pushing the location of maximum width downstream. Nozzle heating has also been shown to significantly influence the nozzle exit region [44].
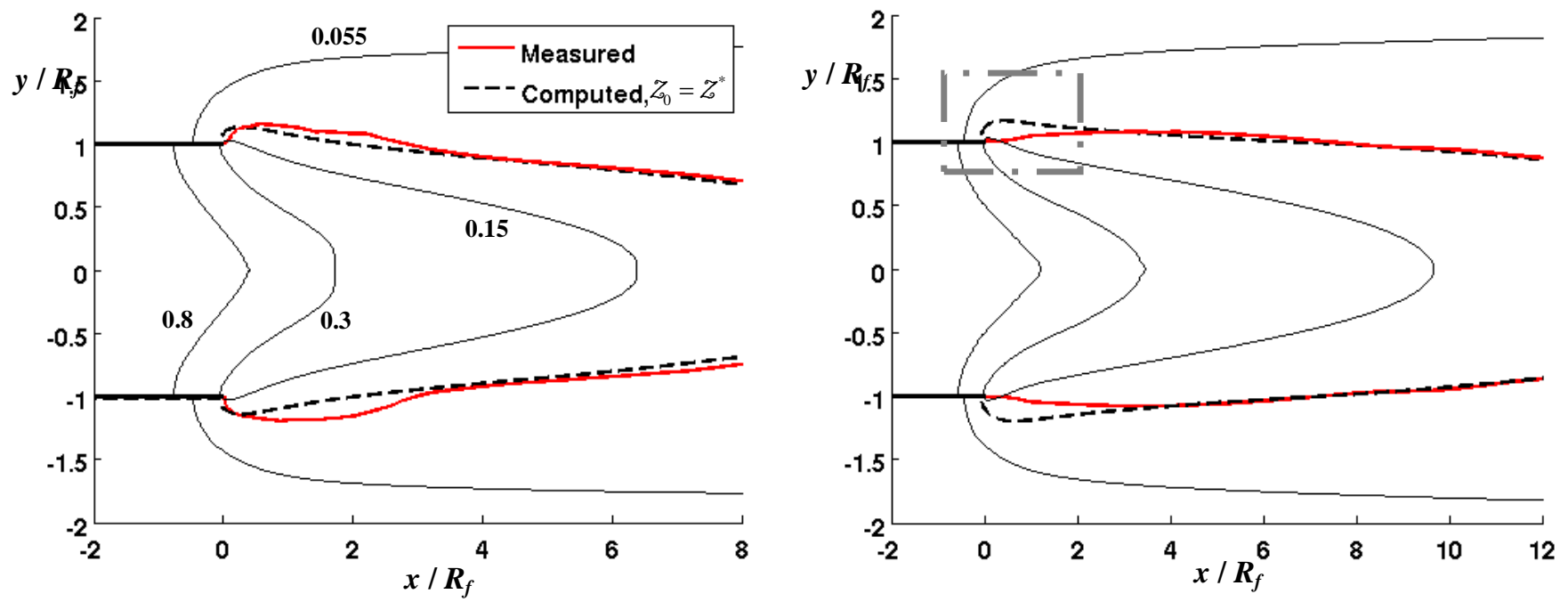

Figure 12 - Experimental and modeled steady state flame position for two representative results for (left) Case $B$ and (right) Case $C$. Also plotted are various computed iso-mixture fraction contours (thin lines) for iso- $Z$ values of $0.055,0.11,0.15,0.3$, and 0.8 . Note the difference in $x$-axis between figures. 
Further downstream, the fact that the flame does not lie on the $Z_{0}=Z_{s t}$ line may reflect some of the other model assumptions; e.g., neglecting radiation effects (which would influence temperature distributions and, therefore the assumed diffusion coefficient field), and differential diffusion effects. Other studies have also analyzed the assumptions required to obtain agreement in mean flame shape between experimental results and computationally extracted iso-contour methods [45, 46]. Again, as mentioned in the opening comments of Section 3, these issues do not create challenges for prediction of premixed flame wrinkle dynamics, as one can simply specify the time averaged flame shape, which is measured.

Given these issues, we adopted the following approach for prediction of the fluctuating flame position - a modified flame identifying mixture fraction value, i.e. $z=z^{*}=0.11$, was utilized for the temperature-diffusion coefficient-mixture fraction relationship, needed for Eq.(8). The steady, $Z_{0}$, and fluctuating, $Z_{1}$, mixture fraction fields were then calculated via, Eq.(9) and Eq.(10), with the use of the spatially varying diffusion coefficient, Eq.(7). Then the mixture fraction properties were determined along the $Z_{0}=Z^{*}$ iso-contour. Of particular interest, which will be discussed in the next section, is the value of $\partial Z_{0} / \partial y$ needed to relate $Z_{1}$ and $\xi_{1}$, which was also calculated along the $Z_{0}=Z^{*}$ isocontour. By following this procedure, we use a consistent approach for calculating $Z_{0}$ and $Z_{1}$, and relating mixture fraction, temperature, and diffusivity. Given the lack of general near-field agreement between the measured flame slope, denoted $\partial \xi_{0} / \partial x$, and that of the $z_{0}=Z^{*}$ iso-contour shown in Figure 12 near $x=0$, we can anticipate discrepancies in the comparison of flame dynamics near $x=0$, an issue we will return to in the next section. 


\section{Space-Time Dynamic Result Comparisons}

This section presents comparisons of the measured flame response characteristics to those predicted from the mixture fraction equation using the measured velocity field as model inputs. While these previously discussed expressions, or computational fields, for $Z_{0}$ and $Z_{1}$ provide solutions for the mixture fraction values over the entire domain, we are particularly interested in the reaction sheet location, which is given by the implicit equation $Z(x, \xi(x, t))=Z_{s t}$. Following the discussion in Section 5.4, an implicit expression for the time averaged flame sheet position, $\xi_{0}(x)$, can be determined from the coordinates where $Z_{0}(x, \xi(x))=Z^{*}$. Following Magina et al.[29], in the limit of small disturbances, an explicit expression for fluctuating flame position can be obtained by expanding the above as:

$$
\xi_{1}(x, t)=-\frac{Z_{1}\left(x, y=\xi_{0}(x), t\right)}{\partial Z_{0} / \partial y\left(x, y=\xi_{0}(x)\right)}
$$

where $\xi_{1}$ is measured in the radial direction, as indicated in Figure 4. Utilizing this expression, flame wrinkle magnitude and phase results can be obtained utilizing the modeled mixture fraction fields, and can be compared to the experimental results for both the left and right flame branches. Some representative comparisons are shown in Figure 13 for various flow and forcing conditions, representative of the range of operational parameters. Note results are only shown for axial extents where measured data exists and the experimental flame was located, hence the shorter axial extent for the lower flow velocity cases. 
Case A
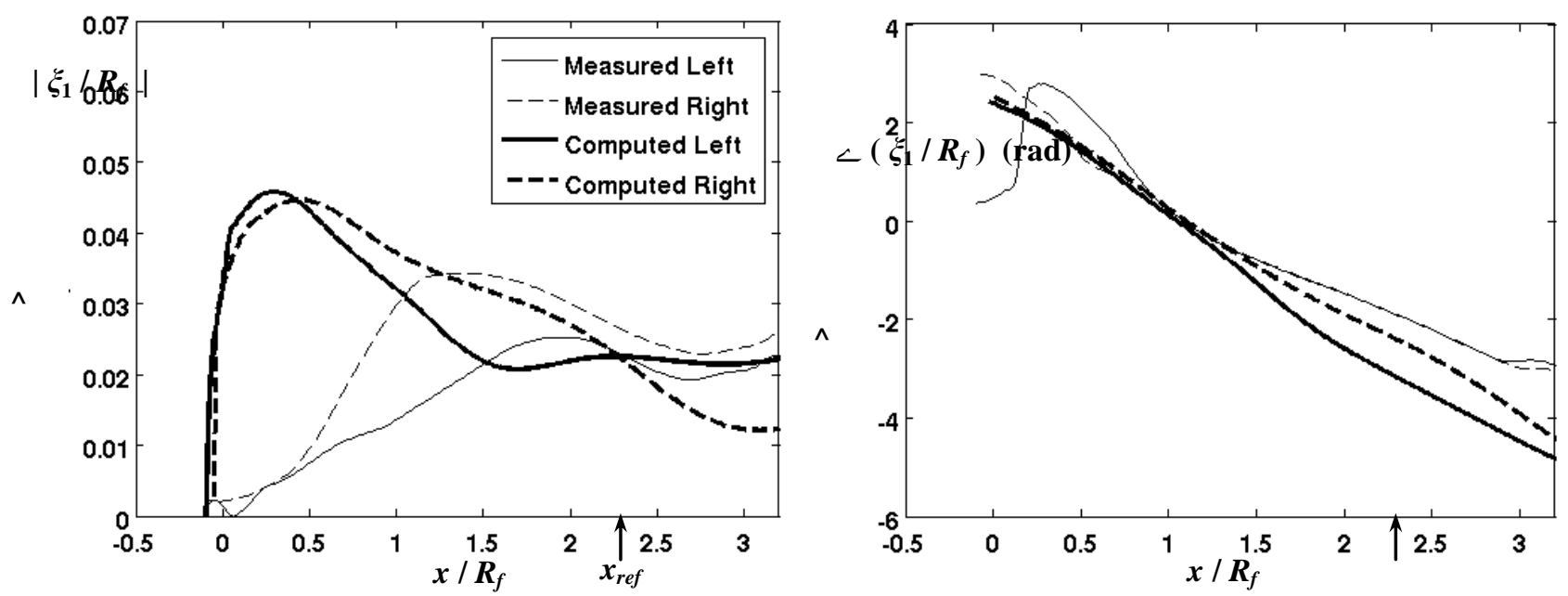

Case B
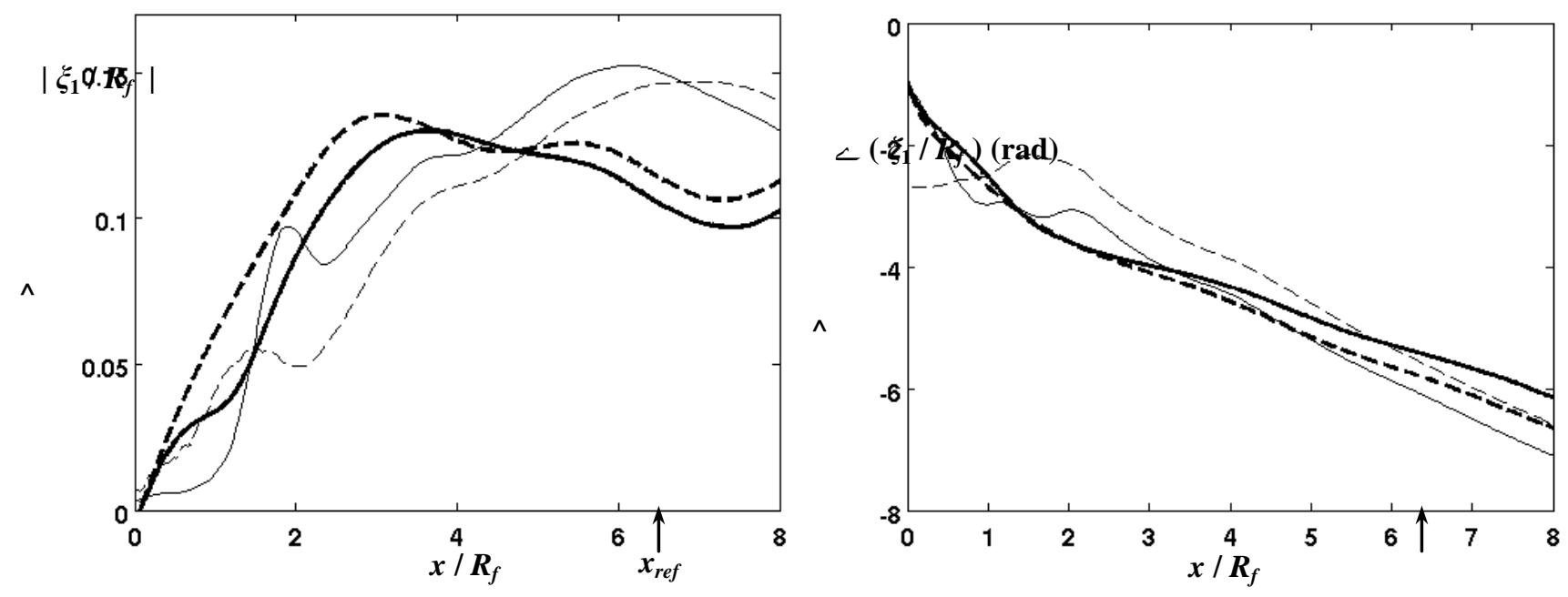

Case C
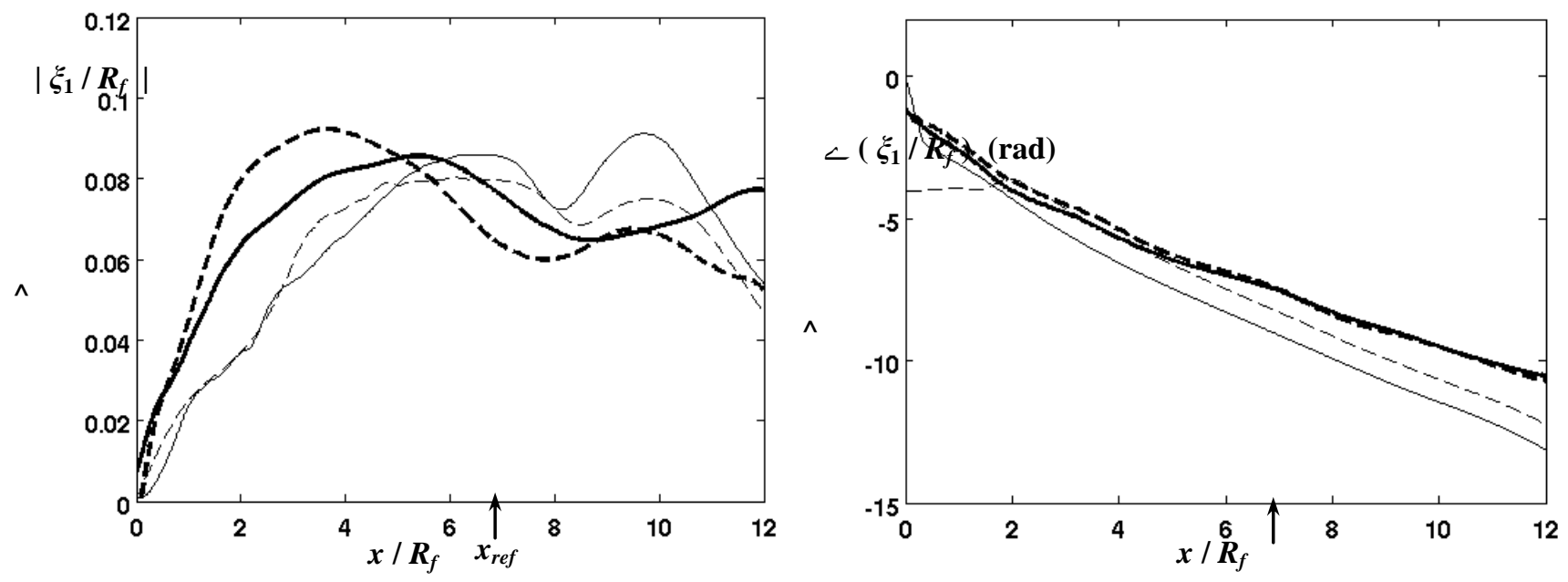


\section{Case D}
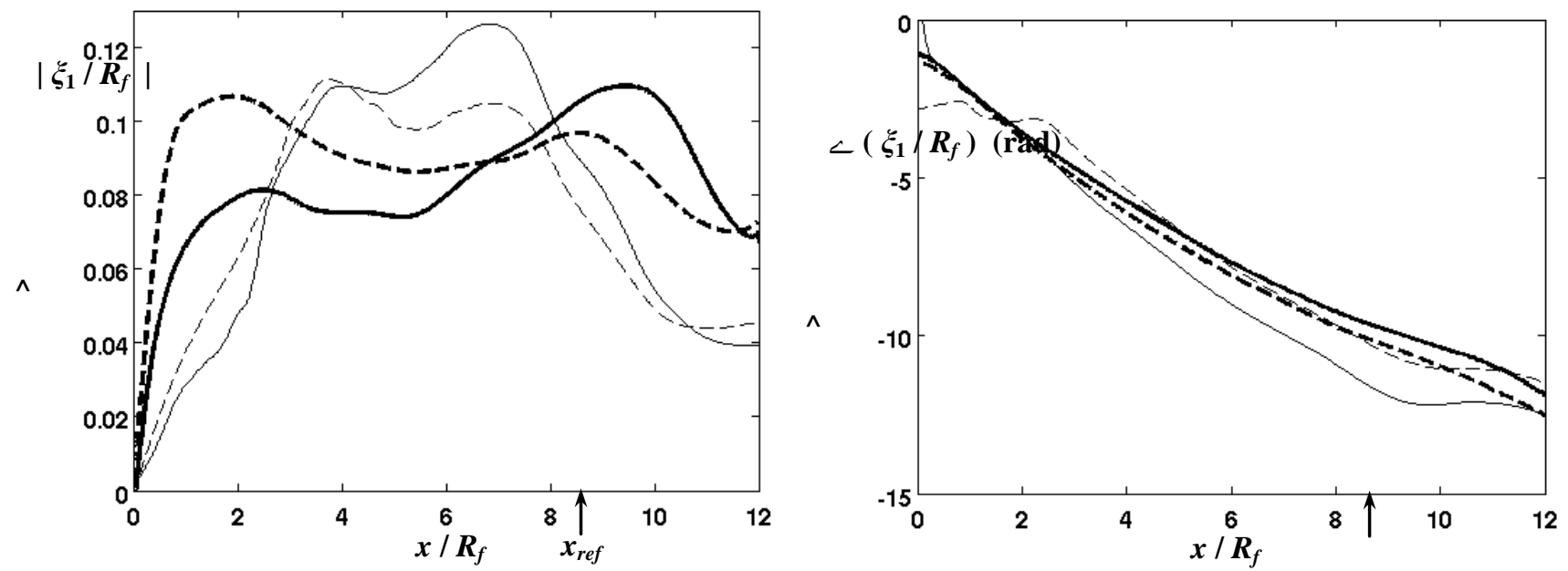

Figure 13 - Representative space-time dynamic magnitude (left column) and phase (right column) comparisons between experimental (measured) and modeling (computed) results for a representative set of data for various $U_{0, \text { des }}$ and $f_{f}$ values. Note: $x_{r e f}$ is denoted for each case in the context of Figure 5.

First, note the qualitative similarities between the experimental and computational results. Specifically, both results predict the rise, peaking and then falloff (possibly accompanied by some modulation) of the amplitude. The values of the peak wrinkle amplitudes are in reasonable agreement with the predictions. Additionally, the phase results also show the near linear rolloff with axial distance, with extremely comparable slopes. However, while the modeled result always captures the general trend of the wrinkle amplitude, the predicted slope of the initial magnitude rise, along with the axial location of the first peaks, does not always compare well to the experiments; for example, in Figure 13, Case B compares much better than Case $\mathrm{C}$ in terms of both initial amplitude rise and peak locations. We will consider this deviation in more detail at the end of this section.

Further downstream, the amplitude shows a maxima/minima pattern caused by wrinkle interference, between flame sheet wrinkles generated at the $x=0$ boundary and disturbances excited locally. The spatial length scale of these maxima depend upon the forcing frequency, with more modulations occurring for higher forcing frequencies, as both expected from the physics and predicted 
by the calculations. With regards to the phase, the calculated curves tend to have more non-linearity than the purely experimental curves, with similar qualitative trends.

The difference in near field dependence of $\left|\xi_{1}\right|$ upon $x$ could be expected from the discussion in Section 5.4, where it was noted that the predicted and measured flame shape also differed near $x=0$. Referring back to Eq.(1) (derived for a much simpler, bulk velocity field), note that this equation can be expanded about $x$ in the near field, and to leading order in $P e$ results in:

$$
\left.\frac{d\left|\hat{\xi}_{1, n}\right|}{d x}\right|_{x=0}=\varepsilon \sin \theta_{0}(x)
$$

Note how this equation directly shows the relationship between the slope of $\left|\xi_{1}\right|$ and $\sin \theta_{0}(x)$ for small $x$. Figure 14 shows how this flame angle quantity was defined, a best fit to the first $0.5 \%$ of the extractable flame extent. In order to evaluate the effect of these near field flame position prediction errors, we define a discrepancy parameter as:

$$
\left.\Delta_{x \sim 0}^{\text {exp-comp }} \equiv(\text { experimental value })\right|_{x \sim 0}-\left.(\text { computed value })\right|_{x \sim 0}
$$

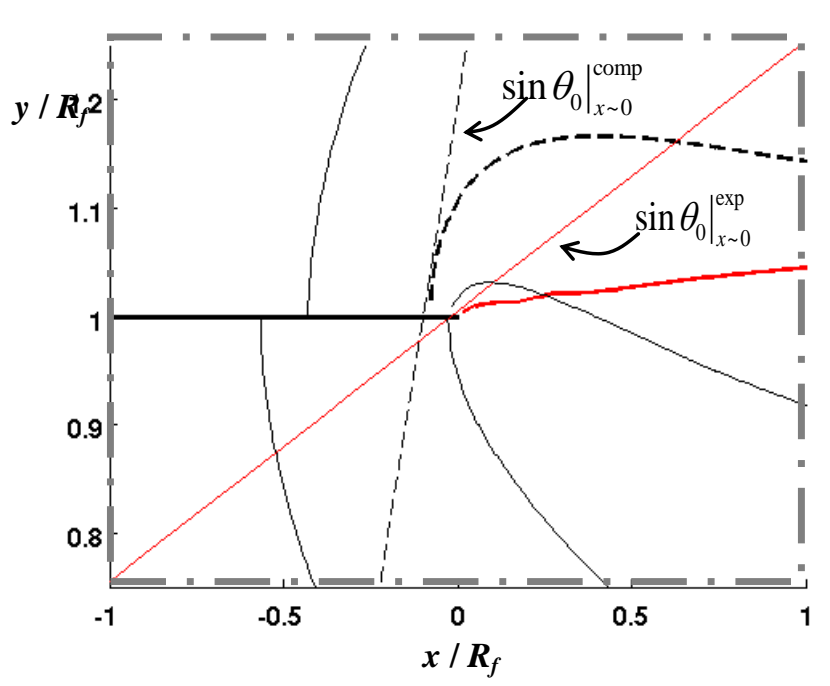

Figure 14 - Zoomed view of boxed region of Figure 12, for Case C, explicitly denoting the $x \sim 0$ computed and experimentally measured flame slopes. 
Figure 15 plots the difference in the experimental and computed wrinkle amplitude rise in the near-base region, $\left|\Delta_{x \sim 0}^{\text {exp-comp }} d\left(\left|\hat{\xi}_{1} / R_{f}\right|\right) / d x\right|$, as a function of the difference in the experimentally extracted and computed time-averaged flame slope, $\left|\Delta_{x \sim 0}^{\text {exp-comp }} \sin \theta_{0}(x)\right|$, for twenty different experimental cases noted in Table I. Notice, how for the cases where the initial flame slope is captured more accurately computationally, the resulting amplitude slope is also more accurate. This result shows the importance in capturing the true near-field mixture fraction field characteristics, and how they are essential to accurately predicting the flame dynamics.

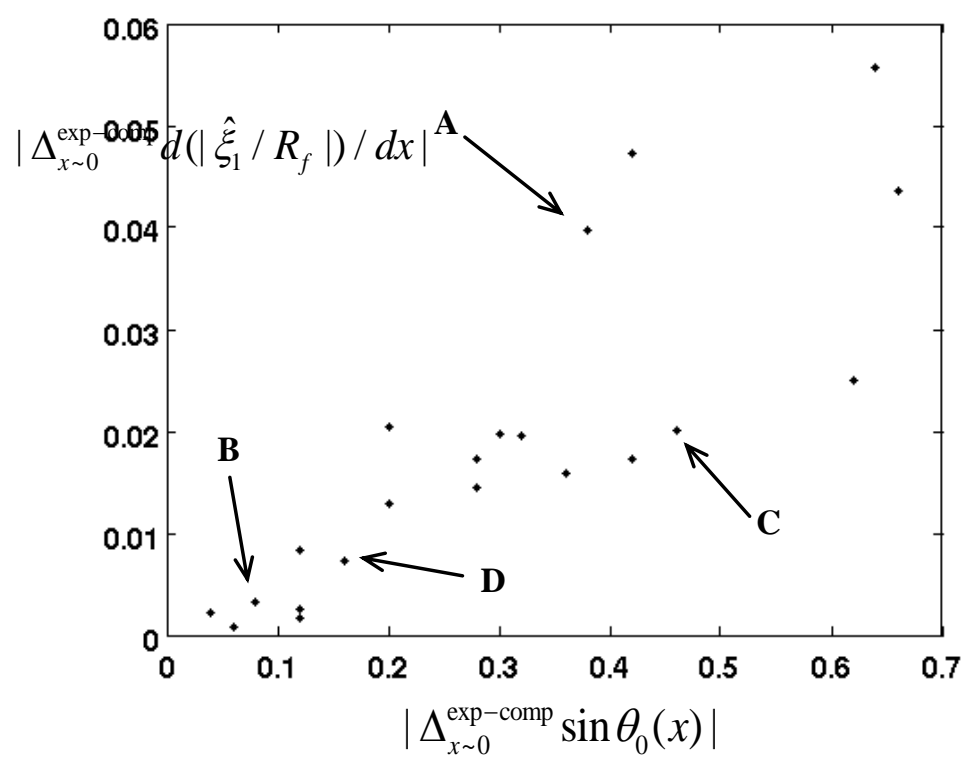

Figure 15 - Plot showing the relation between the difference in the near-base experimental and computed (denoted utilizing $\Delta_{x \sim 0}^{\text {exp-comp }}$ ) flame slope, i.e. $\sin \theta_{0}(x)$, and wrinkle amplitude slope, i.e. $d\left(\left|\hat{\xi}_{1} / R_{f}\right|\right) / d x$, for all edge tractable experimental cases (see Table I). Cases from Table II are explicitly marked.

\section{Concluding Remarks}

An important contribution of these results is corroboration of several earlier predictions on how disturbances on the non-premixed flame sheet evolve in space and time. While these earlier predictions were derived assuming simplified underlying mean and fluctuating disturbance fields, the experimental 
results presented here show several of the same basic phenomenon in real flames. In particular, the results show:

(1) Axial convection of wrinkles on the flamelet; an important implication of this result is that disturbances on the flame sheet are a nonlocal function of the disturbance field. In other words, a wrinkle on the flame sheet at a given spatial location is not only a function of the local flow disturbance, but also the entire upstream spatial distribution of the flow disturbance field.

(2) Near zero magnitude of flame wrinkling at the burner outlet, followed by initial rise in flame wrinkling magnitude. This behavior was previously predicted for flames in the $P e>>1$ and fast chemistry limits. It manifests itself in the anchoring of the non-premixed flame at the burner outlet and is an important distinction between how flames and passive scalars respond to flow disturbances. In the latter case, the magnitude of flame wrinkling would mirror the underlying flow displacement field.

(3) Modulation in the magnitude of flame wrinkling, indicative of interference effects associated with the excitation and axial convection of flame wrinkles. This result is related to (1) above.

To summarize the study results in more detail, a vertical coflowing non-premixed flame facility was developed with axial forcing capabilities. Various diagnostic techniques, such as luminosity and PIV were utilized to experimentally investigate and characterize the spatiotemporal dynamics of the forced non-premixed flame. Utilizing the experimentally measured spatially variant velocity field, along with computed spatially variant diffusion coefficient as model inputs, a revised model was developed for predicting the space-time dynamics of forced diffusion flames. Qualitative agreement was demonstrated between the experimental and modeled results, with regards to flame wrinkle magnitude and phase. Near-base wrinkle amplitude growth, followed by downstream modulatory interference patterns were captured, along with general far-field trends and phase rolloff values. However, not measuring the mixture fraction field as a model input, resulted in some computational limitations, specifically in the near-base region, where the largest differences occurred in terms of the axial dependence of $\left|\xi_{1}\right|$. 
Arising from discrepancies between the modeled and experimental time-averaged flame position, these results emphasize the importance of capturing the appropriate physics essential to the development of a spatio-temporally accurate mixture fraction field, if predictive dynamics are desired. These results could be further enhanced with additional measurements of mixture fraction profiles, as well as directly solving the species equations (to enable incorporation of differential diffusion effects in the computed mixture fractions), and temperature field.

\section{Acknowledgments}

This work has been partially supported by the National Science Foundation under contract CBET1235779, contract monitor, Professor Ruey-Hung Chen.

\section{References}

[1] F. Baillot, D. Durox, R. Prud'Homme, Experimental and theoretical study of a premixed vibrating flame. Combustion and flame, 1992. 88(2): p. 149-152, IN1, 153-168.

[2] S.H. Preetham, T. Lieuwen, Dynamics of laminar premixed flames forced by harmonic velocity disturbances. Journal of Propulsion and Power, 2008. 24(6): p. 1390-1402.

[3] T. Schuller, D. Durox, S. Candel, A unified model for the prediction of laminar flame transfer functions: comparisons between conical and V-flame dynamics. Combustion and Flame, 2003. 134(1-2): p. 21-34.

[4] L. Boyer, J. Quinard, On the dynamics of anchored flames. Combustion and flame, 1990. 82(1): p. 51-65.

[5] T.C. Lieuwen, Unsteady Combustor Physics. 2012: Cambridge University Press.

[6] B. Emerson, U. Mondragon, V. Acharya, D.-H. Shin, C. Brown, V. McDonell, T. Lieuwen, Velocity and flame wrinkling characteristics of a transversely forced, bluff-body stabilized flame, part I: experiments and data analysis. Combustion Science and Technology, 2013. 185(7): p. 1056-1076.

[7] V. Acharya, B. Emerson, U. Mondragon, D.-H. Shin, C. Brown, V. McDonell, T. Lieuwen, Velocity and flame wrinkling characteristics of a transversely forced, bluff-body stabilized flame, Part II: Flame response modeling and comparison with measurements. Combustion Science and Technology, 2013. 185(7): p. 1077-1097.

[8] K. Kim, J. Lee, B. Quay, D. Santavicca, Spatially distributed flame transfer functions for predicting combustion dynamics in lean premixed gas turbine combustors. Combustion and Flame, 2010. 157(9): p. 1718-1730.

[9] D.-H. Shin, T. Lieuwen, Flame wrinkle destruction processes in harmonically forced, laminar premixed flames. Combustion and Flame, 2012. 159(11): p. 3312-3322.

[10] R. Balachandran, B. Ayoola, C. Kaminski, A. Dowling, E. Mastorakos, Experimental investigation of the nonlinear response of turbulent premixed flames to imposed inlet velocity oscillations. Combustion and Flame, 2005. 143(1-2): p. 37-55.

[11] C. Armitage, R. Balachandran, E. Mastorakos, R. Cant, Investigation of the nonlinear response of turbulent premixed flames to imposed inlet velocity oscillations. Combustion and Flame, 2006. 146(3): p. 419-436.

[12] N. Swaminathan, R. Balachandran, G. Xu, A. Dowling, On the correlation of heat release rate in turbulent premixed flames. Proceedings of the Combustion Institute, 2010. 
[13] S. Hemchandra, N. Peters, T. Lieuwen, Heat release response of acoustically forced turbulent premixed flames-role of kinematic restoration. Proceedings of the Combustion Institute, 2011. 33(1): p. 1609-1617.

[14] J. Boulanger, Laminar round jet diffusion flame buoyant instabilities: Study on the disappearance of varicose structures at ultra-low Froude number. Combustion and flame, 2010. 157(4): p. 757-768.

[15] L.-W. Chen, Q. Wang, Y. Zhang, Flow characterisation of diffusion flame in a standing wave. Experimental Thermal and Fluid Science, 2012. 41: p. 84-93.

[16] R. Chrystie, S.H. Chung, Response to Acoustic Forcing of Laminar Coflow Jet Diffusion Flames. Combustion Science and Technology, 2014. 186(4-5): p. 409-420.

[17] S. Dworkin, B. Connelly, A. Schaffer, B. Bennett, M. Long, M. Smooke, M. Puccio, B. McAndrews, J. Miller, Computational and experimental study of a forced, time-dependent, methane-air coflow diffusion flame. Proceedings of the Combustion Institute, 2007. 31(1): p. 971-978.

[18] H.W. Huang, Q. Wang, H.J. Tang, M. Zhu, Y. Zhang, Characterisation of external acoustic excitation on diffusion flames using digital colour image processing. Fuel, 2012. 94: p. 102-109.

[19] B.M. Cetegen, S. Basu, Soot topography in a planar diffusion flame wrapped by a line vortex. Combustion and Flame, 2006. 146(4): p. 687-697.

[20] M. Saito, M. Sato, A. Nishimura, Soot suppression by acoustic oscillated combustion. Fuel, 1998. 77(9): p. 973-978.

[21] S. Dworkin, J. Cooke, B. Bennett, B. Connelly, M. Long, M. Smooke, R. Hall, M. Colket, Distributed-memory parallel computation of a forced, time-dependent, sooting, ethylene/air coflow diffusion flame. Combustion Theory and Modelling, 2009. 13(5): p. 795-822.

[22] Q. Wang, J. Yang, H.W. Huang, Y. Zhang, C. Zhao, Three-dimensional investigation of the dynamics of a propane diffusion flame. Fuel, 2014. 116: p. 448-454.

[23] U. Idahosa, A. Saha, C. Xu, S. Basu, Non-premixed acoustically perturbed swirling flame dynamics. Combustion and Flame, 2010. 157(9): p. 1800-1814.

[24] T.C. Williams, C.R. Shaddix, R.W. Schefer, P. Desgroux, The response of buoyant laminar diffusion flames to low-frequency forcing. Combustion and Flame, 2007. 151(4): p. 676-684.

[25] R.R. Dobbins, M.D. Smooke, A fully implicit, compact finite difference method for the numerical solution of unsteady laminar flames. Flow, turbulence and combustion, 2010. 85(3-4): p. 763-799.

[26] K. Balasubramanian, R. Sujith, Nonlinear response of diffusion flames to uniform velocity disturbances. Combustion science and technology, 2008. 180(1-3): p. 418-436.

[27] M. Tyagi, S. Chakravarthy, R. Sujith, Unsteady combustion response of a ducted non-premixed flame and acoustic coupling. Combustion Theory and Modelling, 2007. 11(2): p. 205-226.

[28] M. Tyagi, N. Jamadar, S. Chakravarthy, Oscillatory response of an idealized two-dimensional diffusion flame: Analytical and numerical study. Combustion and flame, 2007. 149(3): p. 271-285.

[29] N. Magina, D.-H. Shin, V. Acharya, T. Lieuwen, Response of non-premixed flames to bulk flow perturbations. Proceedings of the Combustion Institute, 2013. 34(1): p. 963-971.

[30] N. Magina, V. Acharya, T. Sun, T. Lieuwen, Propagation, dissipation, and dispersion of disturbances on harmonically forced, non-premixed flames. Proceedings of the Combustion Institute, 2015. 35(1): p. 1097-1105.

[31] L. Magri, M.P. Juniper, Sensitivity analysis of a time-delayed thermo-acoustic system via an adjoint-based approach. Journal of Fluid Mechanics, 2013. 719: p. 183-202.

[32] D. Chamberlin, A. Rose, The flicker of luminous flames. Industrial \& Engineering Chemistry, 1928. 20(10): p. 1013-1016.

[33] J. Buckmaster, N. Peters. The infinite candle and its stability-a paradigm for flickering diffusion flames. in Symposium (International) on Combustion. 1988: Elsevier.

[34] S. Ducruix, T. Schuller, D. Durox, S. Candel, Combustion dynamics and instabilities: Elementary coupling and driving mechanisms. Journal of propulsion and power, 2003. 19(5): p. 722-734.

[35] A. Melling, Tracer particles and seeding for particle image velocimetry. Measurement Science and Technology, 1997. 8(12): p. 1406.

[36] C.E. Willert, M. Gharib, Digital particle image velocimetry. Experiments in fluids, 1991. 10(4): p. 181-193. 
[37] J. Soria, A. Masri, D. Honnery. An adaptive cross-correlation digital PIV technique for unsteady flow investigations. in Proceedings of the 1st Australian Conference on Laser Diagnostics in Fluid Mechanics and Combustion, Sydney, NSW, Australia. 1996.

[38] N.A. Magina, T.C. Lieuwen, Effect of axial diffusion on the response of diffusion flames to axial flow perturbations. Combustion and Flame, 2016. 167: p. 395-408.

[39] D. Shin, S. Shanbhogue, T. Lieuwen. Premixed Flame Kinematics in an Axially Decaying, Harmonically Oscillating Vorticity Field. 2008.

[40] J.O. Hirschfelder, R.B. Bird, E.L. Spotz, The Transport Properties for Non-Polar Gases. The Journal of Chemical Physics, 1948. 16(10): p. 968-981.

[41] F.A. Williams, Combustion theory. 2nd ed. 1985, Menlo Park, Ca: Benjamin/Cummings Publishing Co.

[42] R.B. Bird, W.E. Stewart, E.N. Lightfoot, Transport phenomena. 1960. Madison, USA, 1960.

[43] E.N. Fuller, P.D. Schettler, J.C. Giddings, New method for prediction of binary gas-phase diffusion coefficients. Industrial \& Engineering Chemistry, 1966. 58(5): p. 18-27.

[44] Y. Xiong, M.S. Cha, S.H. Chung, Fuel density effect on near nozzle flow field in small laminar coflow diffusion flames. Proceedings of the Combustion Institute, 2015. 35(1): p. 873-880.

[45] R.E. Mitchell, A.F. Sarofim, L. Clomburg, Experimental and numerical investigation of confined laminar diffusion flames. Combustion and flame, 1980. 37: p. 227-244.

[46] T.S. Norton, K.C. Smyth, H.J. Miller, M.D. Smooke, Comparison of experimental and computed species concentration and temperature profiles in laminar, two-dimensional methane/air diffusion flames. Combustion Science and Technology, 1993. 90(1-4): p. 1-34. 